\title{
Front propagation in self-sustained and laser-driven explosive crystal growth: Stability analysis and morphological aspects
}

\author{
Douglas A. Kurtze \\ AT\&T Bell Laboratories, Murray Hill, New Jersey 07974 \\ and Physics Department, Clarkson College of Technology, Potsdam, New York 13676* \\ Wim van Saarloos and John D. Weeks \\ AT\&T Bell Laboratories, Murray Hill, New Jersey 07974
}

(Received 6 February 1984)

\begin{abstract}
We study a simple nonlinear-heat-conduction model for the dynamics of rapid crystallization of amorphous films, and provide a linear stability analysis of the steady-state solutions which describe the propagation with a constant velocity of a straight interface separating the crystalline and amorphous phases. Results are given for the case of self-sustained "explosive" crystallization as well as for the case of cw-laser-driven crystallization. The steady states of the model can have oscillatory instabilities, which result in periodic variations in the amorphous-crystalline interface velocity. For some ranges of the parameters the instability is morphological, so that the interface acquires a wavy shape, while for others it leaves the interface straight. We argue that these two types of instabilities will produce qualitatively different patterns of surface undulations on the crystallized films, similar to those seen in recent experiments on (In, Ga)Sb. Similarities and differences with the MullinsSekerka instability are discussed and the importance of interface kinetics for this instability is pointed out. The onset of the instability is predominantly determined by a parameter related to the activation energy for the amorphous-crystalline transition. Values of this stability parameter are given for $\mathrm{Si}, \mathrm{Ge}$, and $\mathrm{Sb}$. The latter material appears to be close to the threshold for the instability and hence is best suited for an experimental test of our theory.
\end{abstract}

\section{INTRODUCTION}

It is well known that thin films of amorphous materials can be made to crystallize by locally injecting energy with, for instance, a laser pulse or by impact with a stylus. The transition from the metastable amorphous state to the stable crystalline state is accompanied by a release of latent heat, since the crystalline state has the lower entropy. The energy released when some of the material crystallizes can then diffuse to nearby, still-amorphous material, possibly enabling it to crystallize in turn, with the release of more latent heat. Under favorable conditions, a selfsustained avalanche process results, so that once the crystallization has been initiated in some region, the entire film will crystallize. This phenomenon of "explosive" crystallization has been observed in a wide variety of materials.

Experiments have shown ${ }^{1-15}$ that the initial temperature of the film and its substrate must be greater than some critical temperature $T^{*}$ for self-sustained growth to occur. $T^{*}$ depends strongly on the material and on the thickness and history of the film and substrate; some materials, such as $\mathrm{Yb}$ and $\mathrm{Bi},{ }^{16}$ can crystallize explosively at liquid-He temperatures, while $\mathrm{Sb}$ (Refs. 2-6) and (In,Ga)Sb (Refs. 7-9) films exhibit self-sustained crystallization at room temperature and near $100^{\circ} \mathrm{C}$, respectively. When the substrate temperature is slightly above $T^{*}$, experiments by Coffin and Johnston ${ }^{2}$ on $\mathrm{Sb}$ and Wickersham et al..$^{7-9}$ on (In, Ga)Sb have shown that after the film has crystallized, there are often regular, periodic vari- ations in thickness, accompanied by variations in the grain size and possibly in the extent of completeness of the amorphous-crystalline $(a-c)$ transformation. ${ }^{7,8}$ Depending on what the growth conditions were, these undulations may either take the form of parallel "wave fronts" lying perpendicular to the direction of propagation of the crystallization wave, ${ }^{7}$ or of corrugations that exhibit a wavy structure perpendicular to the direction of propagation as well. 8,9 One of our goals in this paper is to provide an explanation for the occurrence of both types of undulations.

At substrate temperatures below $T^{*}$, the latent-heat release will not suffice to sustain the crystallization wave; after initiation it will die out near the triggering area unless additional energy is imparted to the film. In several recent experiments "laser-driven" crystallization in such a case, where the crystallization is maintained by moving a continuous-wave-laser spot at a constant velocity across the film, has been studied. ${ }^{17-26}$

In this paper we provide a theoretical analysis of the macroscopic crystallization dynamics of thin amorphous films, studying both the cases of self-sustained and continuous-wave-laser-driven growth (the well-documented case of laser annealing, ${ }^{27}$ in which a portion of the layer is heated and often melted with a short laser pulse, is not considered here). In accord with the physical picture discussed above, we study heat flow within the film, taking into account the heat produced by the laser, the latent heat generated at the moving $a-c$ boundary, and heat loss to the substrate. A crucial element in determining the fi- 
nal energy balance is the dependence of the crystal-growth rate on the temperature of the $a-c$ boundary due to nonequilibrium interface kinetics. The important role of interface kinetics will be discussed in detail in the next section.

Our work generalizes previous work by Gilmer and Leamy, ${ }^{28}$ Shklovskii, ${ }^{29}$ and van Saarloos and Weeks. ${ }^{30,31}$ Gilmer and Leamy ${ }^{28}$ studied a one-dimensional heatconduction equation incorporating the physical features mentioned above. They found that if the substrate temperature is sufficiently high, then the model indeed has steady-state solutions which describe a straight interface advancing into the amorphous region with a constant velocity. Shklovskii ${ }^{29}$ and van Saarloos and Weeks ${ }^{30,31}$ (hereafter referred to as vSW) analyzed the stability of these self-sustained solutions against small changes in velocity. They showed that for substrate temperatures slightly above critical, and depending on the slope of the growth-rate curve, the steady-state solution can have an oscillatory instability against small velocity perturbations. Numerical analysis of the model equation verified that this instability causes the interface temperature and velocity to oscillate in time. ${ }^{30,31}$ The distance that the model interface moves during one oscillation is of the same order as the wavelength of the experimentally observed surface structure. Since the large velocity changes would naturally be expected to affect details of the crystallization process, vSW argued that the height and composition variations found near $T^{*}$ are induced by this thermal instability.

Since the analysis of vSW was based on a onedimensional model for a straight interface, their work did not address the possibility of morphological instabilities leading to "wavy" structures such as those also observed by Wickersham et al. ${ }^{8,9}$ Here we extend this earlier work by investigating a two-dimensional model of thin-film crystallization. Although we mathematically analyze only the linear stability of possible steady-state solutions, physical arguments and a comparison with the earlier results enable us to obtain a fairly complete picture of the full behavior of the model, even above the instability threshold. We also obtain similar results for cases in which the crystallization is driven by a continuous-wave-laser slit, as in the experiments by Zeiger et al. ${ }^{22}$ discussed below. Our model is essentially that of Gilmer and Leamy ${ }^{28}$ generalized to allow for two-dimensional heat flow in the plane of the film and to include a line energy source (the laser slit).

Previous theoretical work by Zeiger et $a .^{22}$ on laserdriven crystallization neglected the influence of interface kinetics by assuming that the interface remains at a fixed temperature regardless of its velocity. This approximation caused both numerical and physical difficulties in their analysis. Negative interface velocities (corresponding to crystalline material becoming amorphous) arose during the integration of their model equation; this unphysical situation was corrected by artificially setting the velocity to zero whenever the equations made it negative. A proper treatment of interface kinetics avoids these problems automatically. More importantly, it is required for an understanding of the stability of the $a-c$ boundary and is an essential element in the physical mechanism that
vSW proposed to explain the surface undulations.

Temkin and Polyakov, ${ }^{32}$ in an analysis which could be applied to self-sustained crystallization, studied the stability of a planar interface between two phases using a model which included the same physical features as ours (except for the laser) and allowed morphological instabilities. They accounted for the effect of interface kinetics by taking the interface velocity to be a function of interface temperature of the form sketched in Fig. 1(b). This form is appropriate for crystal growth from the melt; as we discuss below, the growth rate for the amorphous-crystalline transition should be given by a curve such as Fig. 1(a). It is clear that the results of Temkin and Polyakov for interfaces operating on the upward-sloping left-hand side of their growth-rate curve are, in principle, applicable to explosive crystallization. However, they made the quasistationary approximation, namely that the temperature field responds instantaneously to changes in the interface position. This approximation is not valid for interfaces moving as quickly as the explosive $a-c$ interface. Indeed, any stability analysis based on it will be unable to detect oscillatory instabilities, since these require that the time evolutions of the interface velocity and of the temperature field be out of phase. In addition, although they correctly located the stability boundary for growth on the right-hand side of the growth-rate curve, and found instabilities for negative interface velocities (i.e., melting), they incorrectly located the stability boundary for growth on the left-hand side of the curve, which is of primary interest to us.

The next section discusses the role of interface kinetics in some detail. Although conceptually straightforward, the linear stability analysis of steady-state solutions is rather tedious, especially because the usual quasistationary approximation is inaccurate. We therefore summarize the main results of this analysis in Sec. III, and discuss their experimental implications there as well. In particular, a comparison with parameters for $\mathrm{Si}, \mathrm{Ge}$, and $\mathrm{Sb}$ is made, and the reason why the thermal instability is predominantly seen in $\mathrm{Sb}$ is clarified. In Sec. IV the extension of the Gilmer-Leamy model to allow for two-dimensional heat flow is discussed and the stability equation is derived.
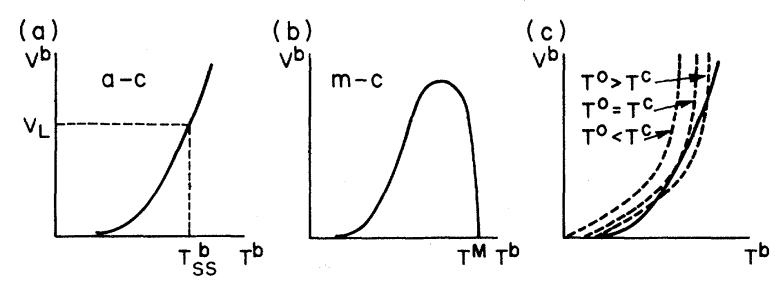

FIG. 1. Dependence of the interface velocity $V^{b}$ on interface temperature $T^{b}$ arising from growth kinetics. (a) Typical Arrhenius curve for the amorphous-to-crystalline transition. For growth driven by a laser slit at a speed $V_{L}, T_{\text {SS }}^{b}$ is the steady-state interface temperature. (b) Typical curve for crystal-melt growth where $T^{M}$ is the equilibrium melting temperature. (c) Growth-rate curve of (a) with the dashed curve of steady-state values $T^{b}=T^{0}+q \sqrt{\beta}$ for explosive crystallization; the intersections of the solid and dashed curves give the possible steady states. The middle dashed curve is for $T^{0}=T^{c}$, the threshold temperature for the existence of steady-state solutions, the left curve is for $T^{0}<T^{c}$, and the right is for $T^{0}>T^{c}$. 
The latter equation is analyzed in detail in Secs. V, VI, and VII for one-dimensional systems, for two-dimensional self-sustained growth, and for two-dimensional laserdriven growth, respectively.

\section{INTERFACE KINETICS}

The growth rate of a crystal is a sensitive function of the temperature because of microscopic kinetics of attachment and detachment of molecules at the interface. The $a-c$ transition is generally an activated process where molecules in "frozen-in" amorphous configurations must surmount an activation barrier to crystallize. Thus the "intrinsic" $a-c$ growth rate of a local region of the $a-c$ boundary can usually be well approximated by an Arrhenius type of behavior,

$$
V^{b} \propto \exp \left(-Q / T^{b}\right),
$$

as sketched in Fig. 1(a) (for data on Si, see, e.g., Ref. 33). Here, $V^{b}$, the velocity with which the crystalline phase grows, is plotted as a function of the temperature $T^{b}$ at the $a-c$ boundary. Note that the growth rate is an increasing function of $T^{b}$.

This behavior is very different from the more familiar case of crystal growth from a slightly undercooled melt. Here the crystal growth rate increases as $T^{b}$ is decreased from the melting temperature $T^{M}$ and, for small deviations, is linear in the undercooling. A typical growth-rate curve for melt growth is drawn in Fig. 1(b). Note that for very low values of $T^{b}$ the curve bends over due to "viscosity effects" and then the behavior resembles that of the $a-c$ system. ${ }^{34-36}$ However, most experiments on directional solidification and dendritic growth occur on the right-hand side of the growth-rate curve, where $T^{b}$ is close to $T^{M}$ and the slope of the growth-rate curve is negative. As pointed out by Shklovskii ${ }^{29}$ and vSW (Refs. 30 and 31 ), this implies that the stability properties of a planar interface growing into an amorphous phase are very different from one growing into a melt. Indeed, the wellknown Mullins-Sekerka and dendritic instabilities ${ }^{37}$ occur only when the slope of the growth-rate curve is negative, as is usually the case for melt growth.

To see this, consider the behavior of a small perturbation along a straight front where part of the boundary bulges forward into the cooler melt [Fig. 2(a)]. The local boundary temperature will drop, which results in an increased driving force for growth [Fig. 2(b)]. Thus the perturbation increases in time [Fig. 2(d)] and the interface can break up into complex patterns (e.g., dendritic) ultimately stabilized by other mechanisms (e.g., surfacetension-like curvature effects). As a result the final length scale of the patterns is the geometric mean of a thermal length and a microscopic (capillary) length.

On the other hand, in the case of the $a-c$ transition [Fig. 2(c)] or of crystal-melt growth on the left-hand side of the growth-rate curve in Fig. 1(b), the growth of the protrusion into the cooler region will slow down with respect to other parts of the interface. Thus we expect stable, although possibly oscillatory, behavior of the $a-c$ interface. The results of the analysis to follow are consistent with these physical considerations.

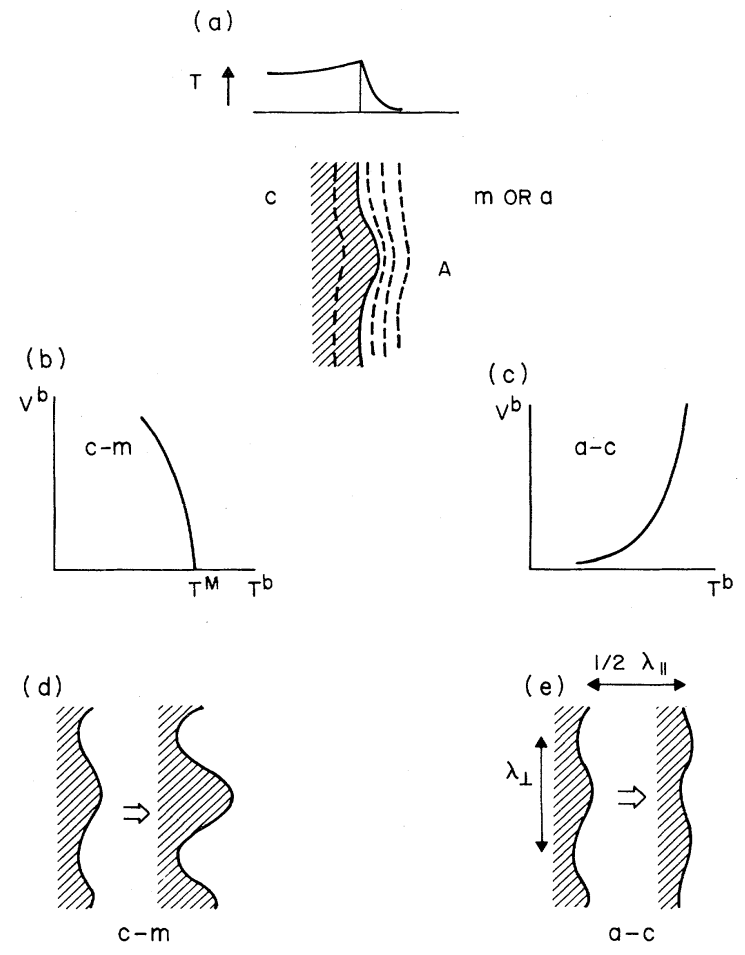

FIG. 2. Evolution of a protrusion on the advancing interface. (a) Protrusion at point $A$ is cooler than the trailing part of the interface. For crystal growth into the melt, the growth-rate curve (b) shows that the interface velocity at $A$ then increases, leading to instability (d). For growth from an amorphous phase, the growth-rate curve (c) shows that the velocity at $\boldsymbol{A}$ decreases, leading to stability or oscillations (e).

\section{SUMMARY OF OUR RESULTS AND DESCRIPTION OF THE IMPORTANT PHYSICAL PARAMETERS IN THE PROBLEM}

The situation that we consider for thin-film crystallization driven by a continuous-wave-laser slit is depicted in Fig. 3. An infinitely long line energy source (the laser slit) of arbitrary profile $J(x)$ is scanned across the film at a constant velocity $V_{L}$ in the $x$ direction. A special case of this analysis, occurring at sufficiently high substrate temperatures, is that of self-sustained growth where the laser is not needed to maintain growth.

In addition to taking into account the temperature dependence of the local $a-c$ growth rate $V^{b}$, we allow a phenomenological dependence of $V^{b}$ on the interfacial curvature. As explained later in this section, we believe that this effect, which is the analog of the GibbsThomson lowering of the equilibrium melting temperature at the crystal-melt interface, ${ }^{37}$ is not strictly necessary in order that the time-dependent propagation fronts stabilize, and indeed our results do not depend sensitively on its magnitude. We find that in order for the model's predictions to make physical sense, the curvature dependence must be such that if the $c$ phase bulges into the $a$ phase, then the interface velocity is lower than for a flat interface. Otherwise, the steady-state solutions of the model would always be unstable against perturbations with suffi- 


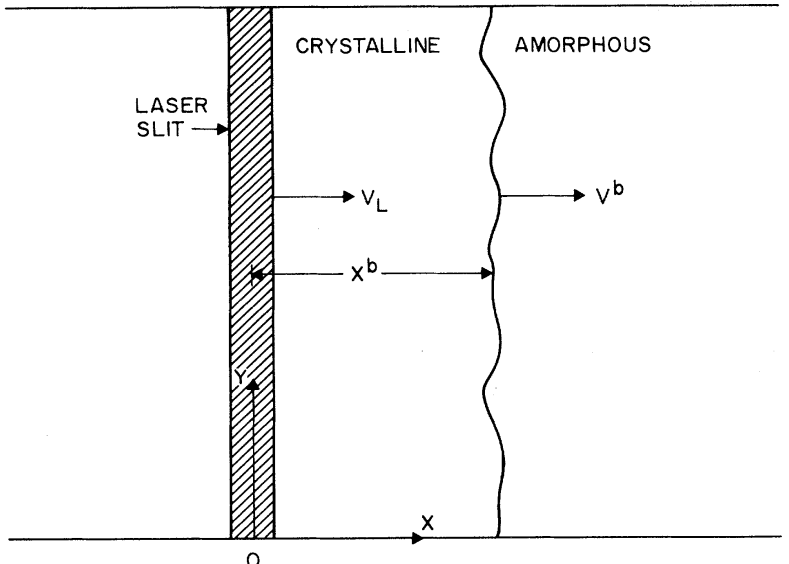

FIG. 3. Schematic view of the laser-driven crystallization of a thin film. The laser slit image, shown crosshatched, defines the $y$ axis; it advances at a constant speed $V_{L}$ in the $x$ direction, driving the amorphous-crystalline interface at $x=x^{b}(y, t)$ ahead of it.

ciently short wavelengths. For the Arrhenius-type $a-c$ growth rate (2.1) the effect of curvature can best be thought of as resulting in an increase of the activation energy. ${ }^{29,32}$

As mentioned above, we consider a two-dimensional version of the Gilmer-Leamy heat-conduction equation ${ }^{28}$ (see Sec. IV for details). In general, we find that our model has steady-state solutions which describe a straight-line $a-c$ interface parallel to the laser slit advancing into the amorphous region at a constant velocity (which equals the laser scan speed $V_{L}$ ). If the laser input power is too small and the substrate temperature $T^{0}$ is too low, then it may not be possible to maintain the interface at a sufficiently high temperature for it to advance at the steady-state velocity $V_{L}$, and continuous crystallization cannot occur. On the other hand, if the substrate temperature is too high, then self-sustained growth at a steady-state velocity $V_{\mathrm{SS}}>V_{L}$ may be possible; in this case the front will outrun the laser. Other cases are possible, but in our discussion of laser-driven growth in this section, we concentrate on the most common experimental situation where substrate temperatures are below $T^{*}$.

We now summarize our main conclusions regarding the stability of these steady-state solutions (assuming that they do exist), based on a linear stability analysis similar to that of vSW, but generalized to allow for fronts with periodic variations with wave number $k$ along the interface; their one-dimensional analysis corresponds to $k=0$ in this work. Details of the analysis are given in Secs. IV-VII.

\section{A. Parameters $\alpha$ and $\beta$}

The stability of self-sustained as well as laser-driven steady-state solutions depends mainly on the value of the parameter

$$
\alpha \equiv \frac{L}{c} \frac{\sqrt{\beta}}{V_{L}}\left[\frac{\partial V^{b}}{\partial T^{b}}\right]_{V^{b}=V_{L}} .
$$

Here $L$ is the latent heat released in the $a-c$ transition, $c$ is the specific heat, ${ }^{38}$ and $\partial V^{b} / \partial T^{b}$ is the derivative of the growth-rate curve $V^{b}=V^{b}\left(T^{b}\right)$ giving the growth rate of the $c$ phase as a function of $T^{b}$ [See Fig. 1(a)]. In the case of self-sustained explosive crystallization, $V_{L}$ should be replaced throughout by the steady-state growth velocity $V_{\text {Ss }}$. The dimensionless parameter $\beta(0 \leq \beta \leq 1)$ is a measure of the importance of heat loss to the substrate. In fact, the combination $L \sqrt{\beta} / c$ is the steady-state increase of the boundary temperature over that of the substrate due to the latent-heat release. (In the presence of a laser, the true steady-state increase of the boundary temperature is the sum of this term and the contribution from the laser.) Hence, $\alpha$ is a dimensionless measure of the steepness of the growth-rate curve. In our model, we find for $\beta$ the explicit result

$$
\beta=V_{L}^{2} /\left(V_{L}^{2}+4 D \Gamma\right),
$$

where $D$ is the thermal diffusivity of the deposited material (denoted $\kappa$ in our earlier work ${ }^{30,31}$ ) and $\Gamma$ is a phenomenological rate constant connected with heat loss to the substrate (see Sec. IV). Note that indeed $\beta \rightarrow 1$ for $\Gamma \rightarrow 0$, as indicated above.

For the Arrhenius-type $V^{b}\left(T^{b}\right)$ dependence (2.1), we obtain for $\alpha$

$$
\alpha=\frac{L}{c} \sqrt{\beta} \frac{Q}{\left(T_{\mathrm{SS}}^{b}\right)^{2}},
$$

where $T_{\mathrm{SS}}^{b}$ is the steady-state boundary temperature. Thus in this case, $\alpha$ is completely determined by experimentally accessible parameters. In particular, if heat loss is negligible, we obtain the simple result

$$
\alpha=\frac{L}{c} \frac{Q}{\left(T_{\mathrm{SS}}^{b}\right)^{2}} \quad(\Gamma \rightarrow 0, \beta \rightarrow 1) .
$$

From our linear stability analysis, we find that the steady-state solutions are unstable when $\alpha$ exceeds a critical value which typically lies between 2 and 4 . We will discuss these instabilities in some more detail in Secs. III B and III C, and then in Sec. III D, we will compare our results with experimental observations on the basis of Eq. (3.4).

\section{B. Self-sustained explosive crystallization}

Our results for the values of the physical parameters at which the steady-state solutions in self-sustained explosive crystallization become unstable do not differ very much from the earlier predictions of vSW for the propagation of a straight front. The result of the linearstability analysis of this paper is depicted in Fig. 4(a), where the parameter $\alpha$, related to the steepness of the slope of the growth-rate curve, is plotted along the horizontal axis and the "heat-loss parameter" $\beta$ is plotted along the vertical axis. In the absence of curvature corrections, the solid and dashed lines mark the stability boundary, i.e., all steady-state solutions having parameter values to the left of these lines are stable.

Note that for a given sample, the possible values of $\alpha$ and $\beta$ characterizing steady-state self-sustained growth are related through the growth-rate curve, so that the 


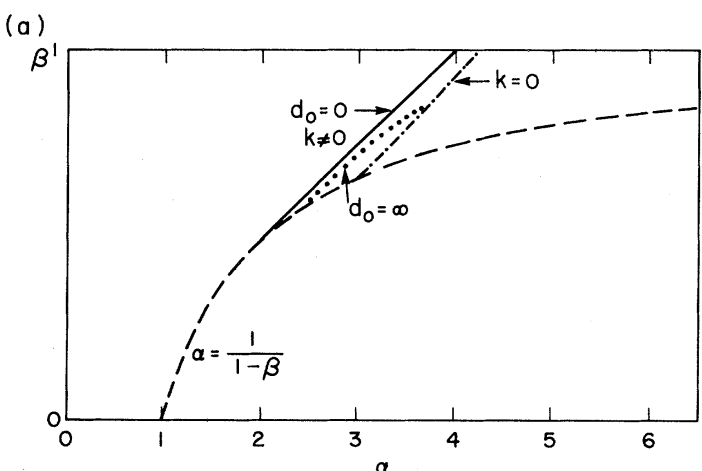

(b)

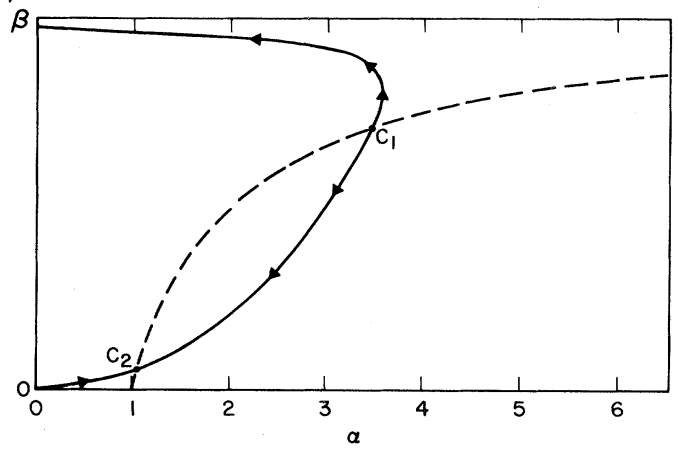

FIG. 4. Stability plot for self-sustained explosive crystallization, with $\alpha$ and $\beta$ defined by Eqs. (3.1) and (3.2). The dashed curve separates always-unstable steady states below it from possibly stable ones above it. (a) Solid and dotted lines are the boundaries at which morphological instabilities occur for zero and infinite capillarity, respectively. (For finite $d_{0}$, the stability boundary lies between these lines.) The dashed-dotted line marks the limit of instability against one-dimensional $(k=0)$ perturbations. Stable steady states lie to the left of the solid $\left(d_{0}=0\right)$ or dotted and dashed-dotted $\left(d_{0}=\infty\right)$ lines. (b) Solid curve represents the possible steady states for a given material. As $T^{0}$ is increased, points representing the stability of the steady states move in the direction shown by the arrows. Apart from a pathological solution at small $\beta$ (cf. Ref. 39), there are no steady-state solutions for $T^{0}<T^{c}$. At $T^{0}=T^{c}$ steady states appear whose stability is represented by a point in (b) near $c_{1}$. The value of $\alpha$ of the steady-state solution whose stability changes [upper branch of the solid line in (b)] decreases for increasing values of $T^{0}$.

steady states lie on a curve in the stability diagram. Such a "material curve" is sketched in Fig. 4(b). Experimentally, one selects a particular steady state by adjusting the substrate temperature $T^{0}$. Theoretically, the steady-state interface temperature and velocity are determined ${ }^{28,31}$ by the intersection of the growth-rate curve and a curve [Fig. 1 (c)] representing steady-state values in the $V^{b}$-versus- $T^{b}$ diagram. For $T^{0}$ below a threshold temperature $T^{c}$, the curves in Fig. 1(c) do not intersect and self-sustained crystallization is not possible. For $T^{0}$ above $T^{c}$ there are typically two intersections, ${ }^{39}$ which merge when $T^{0}$ approaches $T^{c}$ from above. In this limit the stability of the two intersections is represented by points in the stability diagram, Fig. 4(a), approaching the dashed line $\alpha=1 /(1-\beta)$. For $T^{0}>T^{c}$, the lower intersection of Fig. 1 (c) is represented by a point moving down on the materi- al curve from the point $c_{1}$; this steady-state solution is always unstable. The second, interesting steady state, which may be stable or unstable, corresponds to a point on the material curve moving up from $c_{1}$ as $T^{0}$ increases.

In the absence of curvature corrections the stability boundary for the latter steady-state solution is the solid line in Fig. 4(a). To the right of this line, where $\alpha$ exceeds some critical value between 2 and about 4, there is an oscillatory instability just as was found before in the onedimensional model: $T^{b}$ and $V^{b}$ vary periodically in time. However, the new feature of our calculation is that the solid line represents a finite-wave-number oscillatory instability, so that slightly beyond the threshold, one has

$$
V^{b}=V_{0}+V_{1} \sin (\omega t) \sin (k y),
$$

and similarly for $T^{b}$. Note that this line lies to the left of the dashed-dotted line, which represents the instability for straight fronts studied by Shklovskii ${ }^{29}$ and vSW. $^{30,31}$ These solutions represent a "wavy" interface with oscillatory speed and temperature, with different positions $y$ along the interface being out of phase with one another. It is natural to associate the occurrence of this thermal instability with the existence of the wavy structures seen in the experiments of Wickersham et al. ${ }^{8,9}$

The solid line actually represents the stability boundary only in the special case that there is no curvature dependence of the growth rate. In our calculations, we have allowed for such a dependence by introducing the parameter

$$
d_{0} \equiv \frac{-1}{2 D} \frac{\partial V^{b}}{\partial \kappa}
$$

where $\kappa$ is the curvature of the interface, taken to be positive if the $c$ phase bulges into the $a$ phase. Since with this definition $V^{b}$ will decrease with $\kappa$, the arguments given above require that $d_{0}$ be positive.

For increasing values of $d_{0}$, the finite-wave-number instabilities are suppressed more and more toward the dotted line in Fig. 4(a) which represents the case $d_{0}=\infty$, so that for sufficiently large $d_{0}$ the $k=0$ instability line of Shklovskii ${ }^{29}$ and vSW becomes part of the stability boundary. Thus within the context of our model, the $k=0$ instability (describing a straight $a-c$ boundary acquiring an oscillatory speed) will only be observed for large $d_{0}$.

It is physically likely ${ }^{29,32}$ that the curvature dependence of $V^{b}$ enters via a curvature correction to the activation energy. This implies that $d_{0}$ is proportional to $V_{\text {Ss }}$ so that the physically realized stability boundary approaches the dotted $d_{0}=\infty$ line in Fig. 4(a) for large velocities. In this case the $k=0$ line becomes the stability boundary in the upper part of the diagram $(\beta \rightarrow 1) .^{40}$

The physical origin of the oscillatory instability was discussed by vSW. For a boundary moving with an average velocity $V_{\text {av }}$, only the heat released within a distance of the order of $d_{m} \equiv 8 D / V_{\text {av }}$ of a given point can contribute to the temperature at that point. It follows that the wavelength $\lambda_{\|}$of the pattern along the direction of propagation (the distance traveled by the interface during one oscillation) is of the order of $d_{m}$,

$$
\lambda_{\|} \simeq d_{m}=8 D / V_{\mathrm{av}}
$$

Oscillatory velocities can arise because an increase in the 
growth rate will cause the boundary to move ahead so rapidly that the heat diffusion from positions not immediately behind the interface (more than $\frac{1}{4} d_{m}$ away, say) cannot keep up. Then $T^{b}$ and the front velocity drop, after which more heat diffuses to the boundary, so that $T^{b}$ and $V^{b}$ can rise again.

These same physical considerations permit a qualitative understanding of the wavelength $\lambda_{\perp}$ along the interface of the finite- $k$ instability predicted by our analysis. Since in finite- $k$ instabilities the velocities at two positions a distance $\frac{1}{2} \lambda_{\perp}=\pi / k$ apart are out of phase with one another, one expects $\frac{1}{2} \lambda_{\perp}$ to be at least larger than $d_{m} \simeq \lambda_{\|}$. We have calculated the aspect ratio $\lambda_{\|} / \lambda_{\perp}$ for the mode that first becomes unstable as a function of $d_{0}$ and $\beta$. For parameter values close to the stability boundary, this mode will dominate the oscillatory component of the solution. The results are plotted in Fig. 5 and confirm our argument that the aspect ratio should typically be of the order of $\frac{1}{2}$, although large values of $d_{0}$ can suppress the aspect ratio below this value.

Although it does not follow directly from the linear stability analysis, it should be clear from the above discussion that one generally expects the amplitude of the wavy structure along the interface to be less than $d_{m}$, because otherwise it would not be possible for points bulging forward into the $a$ phase to move temporarily faster and thus to have a somewhat higher boundary temperature. For this reason, and because the aspect ratio stays finite in the limit $d_{0} \rightarrow 0$, we believe that it is not necessary for the stability of the moving boundary to take the curvature dependence of the growth rate into account via the parameter $d_{0}$. This indicates that an expansion involving only a few $k$ modes would suffice for an analysis of the nonlinear crescentlike patterns observed by Wickersham et al. ${ }^{8,9}$ In contrast, the Mullins-Sekerka instability on the right-hand side of the growth-rate curve is suppressed at short wavelengths only by such capillary effects. As a

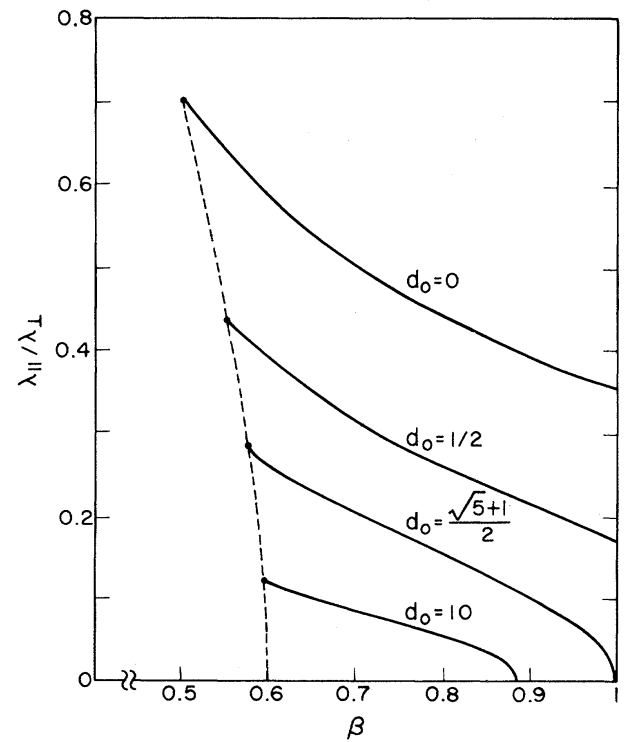

FIG. 5. Aspect ratio $\lambda_{\|} / \lambda_{\perp}$ for self-sustained growth near the morphological instability curve. The dashed curve marks the appearance of morphological instabilities with $\alpha=1 /(1-\beta)$. result the dendrite-like patterns beyond this instability require a much more complex treatment.

\section{Stability of laser-driven steady-state solutions}

We now briefly discuss the stability of laser-driven steady-state solutions. The presence of the laser introduces another parameter,

$$
R \equiv \frac{-2 D c}{L V_{L} \sqrt{\beta}}\left(\frac{d T_{L}}{d x}\right)_{x=x^{b}},
$$

where $T_{L}(x)$ is the temperature profile introduced by the laser and whose derivative is evaluated at the steady-state interface position $x^{b}$. To understand the meaning of this parameter, we note that for $\beta \simeq 1$, we may write ${ }^{41}$

$$
R \simeq 2\left(\frac{d T_{L}}{d x}\right)_{x=x^{b}}\left(\frac{d T_{\mathrm{th}}}{d x}\right)_{x=x^{b}+}^{-1},
$$

where $d T_{\text {th }} / d x$ is the derivative of the steady-state temperature profile induced by the latent heat alone and evaluated just in front of the interface. This derivative is usually quite large and under most experimental conditions greater than or of the order of $d T_{L} / d x$. Hence, $R$ is typically of order unity.

From the linear stability analysis, we find that for $R \lesssim 1$, the stability diagram does not differ qualitatively from the one given above for self-sustained explosive crystallization, although the onset of the instability is shifted towards larger values of $\alpha$ for increasing $R$ (for explicit results, see Fig. 6). In analogy with the oscillations occurring for self-sustained explosive crystallization for small damping, this leads to the prediction that the laser-driven steady-state propagation becomes unstable when $\alpha$ exceeds some threshold value around 4. Slightly beyond the threshold, the interface will oscillate periodically back and forth on the steep side of the laser profile.

To control the parameters experimentally, note that the values of $\alpha$ and $\beta$ appropriate to a laser-driven steady

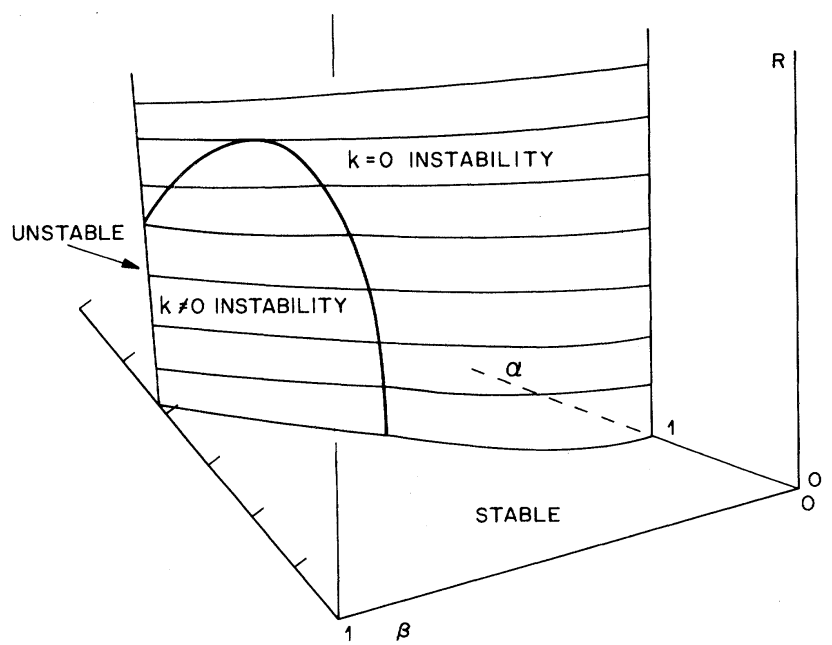

FIG. 6. Stability plot for laser-driven crystallization for small but finite capillarity $d_{0}$, with $\alpha, \beta$, and $R$ defined in (3.1), (3.2), and (3.8). Steady states represented by points in front of the surface (smaller $\alpha$ ) are linearly stable. 
state are related through the growth-rate curve, as in the case of self-sustained growth discussed above. In the laser-driven case, however, their values are set by the laser velocity $V_{L}$ rather than the substrate temperature: $V_{L}$ determines $\beta$ directly via Eq. (3.2) and also fixes the operating point on the growth-velocity curve as illustrated in Fig. 1(a), thus determining $\alpha$. Note that $\alpha$ and $\beta$ still lie on the same curve as for self-sustained growth. In general, from Eq. (3.9), $R$ is increased by decreasing the substrate temperature. However, if the laser power is so low that the interface is actually within the area illuminated by the laser, then it will also affect the value of $R$. If, on the other hand, it is sufficiently high that the interface is not actually within the illuminated area, then the laser temperature profile $T_{L}(x)$ is exponential at the interface, so that $R$ will simply be proportional to $T_{L}\left(x^{b}\right)$, which is completely determined by $V_{L}$ and the substrate temperature. Thus, $R$ will be independent of the laser power, and the theoretical parameters $\alpha, \beta$, and $R$ will depend only on the laser velocity and substrate temperature.

\section{Comparison with experiments}

As discussed above, under most experimental conditions our theory predicts that steady-state solutions will be unstable when the stability parameter $\alpha$ exceeds some value between about 2 and 4. In Table I we estimate typical values of $\alpha$ for $\mathrm{Si}, \mathrm{Ge}$, and $\mathrm{Sb}$. Although the activation energy $E_{a}$ of $\mathrm{Sb}$ is not known very precisely, Table I shows that $\mathrm{Sb}$ is likely to be roughly in the range where the instability sets in and where it should be possible to see the oscillations predicted by our theory most clearly. It is therefore reasonable to associate the periodic variations in Sb films seen by Coffin and Johnston ${ }^{2}$ with the occurrence of this instability. Unfortunately, we have been unable to find data for $L / c$ and $E_{a}$ of $\mathrm{GaSb}$ or (In,Ga)Sb, the materials for which Wickersham et al. ${ }^{7-9}$ observed both the parallel and the wavy surface undulations. If the occurrence of these structures is indeed associated with a value of $\alpha$ of the order of 3, then (In, Ga)Sb should have a rather low activation energy for the $a-c$ transition or (less likely) a small value of $L / c$.

Experimentally, the surface roughness is found to de- crease for increasing values of $T^{0}$. vSW associated this behavior with a leveling off of the growth-rate curve. A much more convincing explanation, however, is based on the observation that the stability parameter $\alpha \propto\left(T^{b}\right)^{-2}$ decreases for increasing values of $T^{b}$ and hence $T^{0}$ [cf. Eq. (3.3) and Fig. 4(b)]. Therefore, an increase in $T^{0}$ will, in general, result in a decrease in the amplitude of the oscillations and, eventually, a stabilization of the steady-state growth.

Table I also shows that $\mathrm{Si}$ and Ge have a large $\alpha$ and hence are far in the unstable regime; thus steady-state growth of the $c$ phase will normally be impossible for these materials. Although we have not performed detailed numerical calculations to investigate what would happen this far in the unstable nonlinear regime, it is clear that in such cases our model would show that the $a-c$ boundary would quickly speed up at first, then outrun the laser beam, and finally stop when it lacks the support from the laser heat (experimental conditions are such that self-sustained growth is not possible). Crystallization finally starts again when the laser catches up to the boundary region. Such a behavior would be consistent with the experimental observations by Auvert et al. ${ }^{25-27}$ on $\mathrm{Si}$ as well as with those of Zeiger et al..$^{22}$ on Ge, and is similar to what Zeiger et al. ${ }^{22}$ found in their theory, which may be viewed as the $\alpha \rightarrow \infty$ limit of our work.

It must be kept in mind, however, that because of the large activation energy, the direct $a-c$ transition in Ge is never rapid enough, even at boundary temperatures of $1000 \mathrm{~K}$, to give the experimentally observed growth rates of the order of meters per second. Thus Gilmer and Leamy ${ }^{22}$ suggested that there probably is a liquid zone in between the $a$ and $c$ phases ( $a$-Ge is believed to melt at about $970 \mathrm{~K}$ ). This prediction was verified by Leamy et $a l .{ }^{42}$ The crystalline Ge then grows from a highly undercooled melt, probably on the right-hand side of the growth-rate curve [Fig. 1(b)], thus giving the possibility of Mullins-Sekerka-type instabilities rather than those studied in this paper. The situation for $\mathrm{Si}$ is somewhat less clear-although $a$-Si has a melting temperature $T_{a}^{M}$ somewhere between 1335 and $1460 \mathrm{~K},{ }^{43}$ Auvert et al. apparently have sometimes observed direct $a-c$ transitions with rather large growth rates near these temperatures,

TABLE I. Experimental values for various parameters in the definition of the stability parameter $\alpha$. The values of $T^{b}$ for $\mathrm{Sb}$ and $\mathrm{Ge}$ were estimated as the sum of $L / c$ and the substrate temperature used in Refs. 2-6 and 49, respectively, and therefore tend to be too large in view of the neglect of heat losses. The value of $1300 \mathrm{~K}$ is taken for Si because $T^{b}$ needs to be of this order to obtain growth rates of centimeters per second.

\begin{tabular}{lcccccrr}
\hline \hline & $\begin{array}{c}L \\
(\mathrm{cal} / \mathrm{g})\end{array}$ & $\begin{array}{c}c \\
(\mathrm{cal} / \mathrm{g} \mathrm{K})\end{array}$ & $\begin{array}{c}L / c \\
(\mathrm{~K})\end{array}$ & $\begin{array}{c}E_{a} \\
(\mathrm{eV})\end{array}$ & $\begin{array}{c}Q=E_{a} / k_{B} \\
(\mathbf{K})\end{array}$ & $\begin{array}{c}T^{b} \\
(\mathbf{K})\end{array}$ & $\alpha$ \\
\hline $\mathrm{Si}$ & $84^{\mathrm{a}}$ & $0.2^{\mathrm{b}}$ & 420 & $2.7^{\mathrm{c}}$ & 31300 & 1300 & 7.8 \\
$\mathrm{Ge}$ & $40^{\mathrm{d}}$ & $0.09^{\mathrm{b}}$ & 444 & $1.5^{\mathrm{e}}$ & 17400 & 900 & 9.5 \\
$\mathrm{Sb}$ & 20 & $0.06^{\mathrm{b}}$ & 333 & $0.09-0.27^{\mathrm{f}}$ & $1040-3130$ & 600 & $1-2.9$ \\
\hline \hline
\end{tabular}

${ }^{2}$ Reference 43.

${ }^{\text {b}}$ Reference 47.

${ }^{\mathrm{C}}$ Reference 33.

${ }^{\mathrm{d}}$ Reference 46.

${ }^{\mathrm{e}}$ Reference 48.

${ }^{\mathrm{f}}$ Reference 6. 
while Thompson et $a l .{ }^{44}$ find evidence for the presence of a liquid layer. In $\mathrm{Sb}$, on the other hand, the experiments of Bostanjoglo and Schlotzhauer ${ }^{6}$ gave no evidence for the presence of a liquid zone. ${ }^{45}$

Thus, if it is possible to find the direct $a-c$ transition (i.e., no liquid zone) in laser-driven explosive crystallization of high-activation-energy materials, this occurs far in the unstable regime. Lower-activation-energy materials, such as $\mathrm{Sb}$, however, can be close to the threshold and should show the behavior discussed above. Hence, these are more appropriate candidates for an experimental test of our ideas, using, e.g., the time-resolved transmissionelectron-microscopy (TEM) technique of Bostanjoglo and Schlotzhauer. ${ }^{6}$

\section{THE MODEL AND ITS STABILITY EQUATION}

To model the laser-driven crystallization process, we think of a laser slit, which defines the $y$ direction, being moved at a constant velocity $V_{L}$ perpendicular to itself in the $x$ direction as sketched in Fig. 3. We will work in a frame of reference moving with the laser. The power density provided by the continuous-wave laser then depends only on $x$, and we denote it by $J(x)$. The $a-c$ interface is located at $x=x^{b}(y, t)$ relative to the laser. We consider only heat diffusion in the film, and assume that the film is sufficiently thin that the diffusion is essentially twodimensional. Heat loss to the environment (including the substrate) is treated crudely using a phenomenological damping term. In the moving frame of reference, heat diffusion in the layer is described by

$$
\begin{aligned}
\frac{\partial T}{\partial t}= & D \nabla^{2} T+V_{L} \frac{\partial T}{\partial x}-\Gamma\left(T-T^{0}\right) \\
& +\frac{J(x)}{c d}+q V^{b} \delta\left(x-x^{b}(y, t)\right) .
\end{aligned}
$$

Here $T(x, y, t)$ is the temperature of the layer, $D$ is its thermal diffusivity and $c$ is its volumetric specific heat, both of which we take to be the same in the amorphous and crystalline phases, ${ }^{38} d$ is the thickness of the layer, $q=L / c$, where $L$ is the latent heat of crystallization, $T^{0}$ is the substrate temperature, and $\Gamma$ is a phenomenological constant which accounts for heat loss to the environment. Finally,

$$
V^{b}=V_{L}+\frac{\partial x^{b}}{\partial t}
$$

is the velocity of the interface relative to the substrate. As discussed above, we assume that this interface velocity depends on the local boundary temperature and curvature,

$$
V^{b}=V^{b}\left(T\left(x^{b}(y, t), y, t\right), \kappa\right)=V^{b}\left(T^{b}, \kappa\right),
$$

where the graph of $V^{b}$ versus $T^{b}$ has the general form of Fig. 1(a), and

$$
\kappa=-\frac{\partial^{2} x^{b}}{\partial y^{2}}\left[1+\left[\frac{\partial x^{b}}{\partial y}\right]^{2}\right]^{-3 / 2}
$$

is the curvature of the interface, defined to be positive when the crystalline region bulges into the amorphous region.
A simple steady-state solution to this problem is one in which the interface is a straight line parallel to the laser slit, moving at a constant speed, which must be $V_{L}$, with respect to the substrate. This solution is

$$
\begin{aligned}
& x^{b}(y, t)=x_{\mathrm{SS}}^{b}=\mathrm{constant}, \\
& T(x, y, t)=T_{\mathrm{SS}}(x)=T^{0}+T_{L}(x)+q V_{L} G_{\mathrm{SS}}\left(x-x_{\mathrm{SS}}^{b}\right),
\end{aligned}
$$

where

$$
\begin{aligned}
G_{\mathrm{SS}}(x)= & \left(V_{L}^{2}+4 D \Gamma\right)^{-1 / 2} \\
& \times \exp \left[-\frac{V_{L}}{2 D} x-\frac{\left(V_{L}^{2}+4 D \Gamma\right)^{1 / 2}}{2 D}|x|\right]
\end{aligned}
$$

is the steady-state Green's function for diffusion in the $x$ direction in the moving frame, and

$$
T_{L}(x)=\frac{1}{c d} \int_{-\infty}^{\infty} G_{\mathrm{SS}}\left(x-x^{\prime}\right) J\left(x^{\prime}\right) d x^{\prime}
$$

is the part of the temperature field due entirely to the laser. ${ }^{50}$ The interface position $x_{\mathrm{SS}}^{b}$ is determined by the self-consistency requirement that the interface velocity $V^{b}$ must be equal to $V_{L}$. To find it, we must first solve

$$
V^{b}\left(T_{\mathrm{SS}}^{b}, \kappa=0\right)=V_{L}
$$

for the steady-state interface temperature $T_{\mathrm{SS}}^{b}=T_{\mathrm{SS}}\left(x_{\mathrm{SS}}^{b}\right)$ as shown in Fig. 1(a), and then, from Eq. (4.5b), solve

$$
T_{L}\left(x_{\mathrm{SS}}^{b}\right)=T_{\mathrm{SS}}^{b}-T^{0}-q \sqrt{\beta}
$$

for $x_{\mathrm{SS}}^{b}$, as shown in Fig. 7, with $\beta$ defined in Eq. (3.2). As we can see from Fig. 7 , there can be no steady state if $T^{0}$ is either so low that $T_{\mathrm{SS}}^{b}-T^{0}-q \sqrt{\beta}$ is greater than the maximum value of $T_{L}(x)$ or so high that $T_{\mathrm{SS}}^{b}-T^{0}-q \sqrt{\beta}$ is negative. In the former case there simply is not enough energy being fed into the system to crystallize the sample completely at the desired rate. In the latter, there is a steady-state self-sustained solution in which the interface moves faster than $V_{L}$, so that it runs away from the laser.

When conditions are such that a laser-driven steady

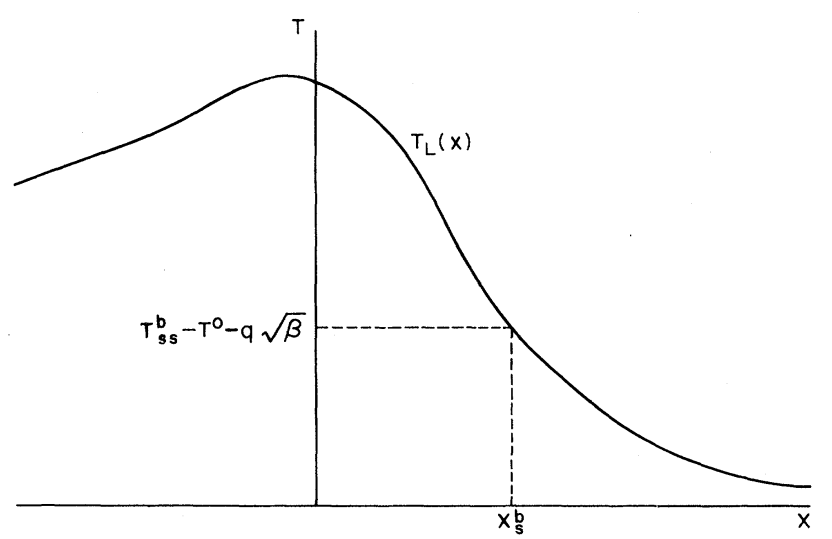

FIG. 7. Temperature profile $T_{L}(x)$ produced by the laser. The steady-state interface position $x_{\mathrm{SS}}^{b}$ is found by solving Eq. (4.9), where $T_{\text {SS }}^{b}$ is found from the growth-rate curve as shown in Fig. 1(a). 
state exists, we investigate its stability by calculating the evolution of an infinitesimal perturbation of the form

$$
\begin{aligned}
& x^{b}(y, t)=x_{\mathrm{SS}}^{b}+\epsilon \exp \left[i \frac{V_{L}}{2 D} k y+\frac{V_{L}^{2}}{2 D} \omega t\right], \\
& T(x, y, t)=T_{\mathrm{SS}}(x)+\epsilon \hat{T}(x) \exp \left[i \frac{V_{L}}{2 D} k y+\frac{V_{L}^{2}}{2 D} \omega t\right] .
\end{aligned}
$$

Here, $k$ and $\omega=\omega\left(k^{2}\right)$ are the dimensionless wave number and growth rate of the perturbation. As shown in Appen$\operatorname{dix} \mathbf{A}, \omega$ must satisfy

$$
\left[\omega+\alpha(1+R)+d_{0} k^{2}\right]\left(1+\beta k^{2}+2 \beta \omega\right)^{1 / 2}=\alpha(\omega+1),
$$

where the square root has a positive real part and the dimensionless quantities $\alpha, \beta, d_{0}$, and $R$ are defined in Eqs. (3.1), (3.2), (3.6), and (3.8). The quantity $\beta$, which lies between 0 and 1, measures the importance of heat loss: $\beta$ near 1 means small $\Gamma$ or large $V_{L}$. The slope of the growth-velocity curve at steady state is given by $\alpha$. Similarly, $d_{0}$ measures the change in interface velocity due to curvature of the interface. We have argued above and will verify below that $d_{0}$ should be positive for our choice of the sign of $\kappa$. Finally, $R$ measures the slope of the laser temperature profile at the interface; it will also be positive. If there is any $k$ for which $\omega\left(k^{2}\right)$ satisfying the stability equation (4.11) has a positive real part, then the steady state is unstable.

At this point it is worthwhile to stress the distinction between the stability boundary, which is the surface in $\alpha$ $\beta-R$ space at which the steady-state solution becomes unstable, and the critical surface (or critical curve in the $\alpha-\beta$ plane, when $R$ is zero) for a given $k$, at which the steadystate solution, which may already be unstable against perturbations with other wave numbers, becomes unstable against those with the given $k$. Critical surfaces are located by simply setting $\operatorname{Re}(\omega)=0$ in the stability equation and eliminating $\operatorname{Im}(\omega)$ with $k$ held fixed, while the stability boundary is the envelope of the set of critical surfaces for all $k$.

If we square both sides of the stability equation, we obtain a cubic polynomial in $\omega$ with real coefficients. Thus there are at most three solutions or branches of $\omega\left(k^{2}\right)$, corresponding to modes of disturbance of the interface which decay or grow linearly at short times. These modes can include at most one oscillatory mode, which would show up as a pair of complex-conjugate solutions.

Note that the only feature of the laser temperature profile which appears in the stability equation (4.11) is $R$, a measure of its gradient at the interface. Setting $R=0$ is then equivalent to considering a problem in which the laser is absent, which is that of self-sustained crystallization, provided that $V_{L}$, which appears in the definitions of $\alpha, \beta$, and $d_{0}$, is replaced by $V_{\mathrm{Ss}}$, the steady-state velocity of explosive growth. The steady-state problem has a symmetry which is destroyed when $R$ is nonzero, namely that the solution is unaffected if the entire system is translated in the $x$ direction. This symmetry is manifested by the fact that when $R$ vanishes, the stability equation is satisfied by $\omega=0$ with $k=0$. In fact, it is easy to see that when $\alpha$ and $d_{0}$ are positive, the stability equation will allow $\omega=0$ only when $R=0$. In this case, one of the three possible modes is the translation mode. In addition, another mode has $\omega$ real and increasing through 0 as $\alpha$ increases through $1 /(1-\beta)$, which then forms part of the stability boundary for the one-dimensional self-sustained situation. As pointed out in Sec. III, this part of the stability boundary is not of great importance, as it separates two different types of steady-state solutions that may exist, one of which is always unstable. ${ }^{30,31}$ The interesting one that may be stable or unstable is represented in the stability diagram by points to the left of the curve $\alpha=1 /(1-\beta)$. If $k$ or $R$ is increased from zero, the linear growth rate of the first unstable mode will acquire an imaginary part and so the mode will be oscillatory.

As we have discussed before (Sec. III) the parameter $d_{0}$ must be positive (or zero) for physical reasons. One can in fact show explicitly from Eq. (4.11) that if $d_{0}$ were negative, all steady-state solutions would become unstable against short-wavelength perturbations. The reason for this is that if the interface curves, then the part having positive curvature, i.e., where the crystalline region bulges into the amorphous region, is also farther from the laser. The usual stabilizing effect is still present: Since this leading part of the interface is farther from the laser, it becomes cooler than the rest of the interface and so tends to slow down, thus allowing the trailing part of the interface to catch up to it. However, if $d_{0}$ were negative, then the positive curvature would increase the growth velocity of the leading part of the interface. This effect is destabilizing, and for sufficiently large curvatures or short wavelengths it would overcome the stabilizing decrease in velocity due to the lowered interface temperature. Thus negative $d_{0}$ would make the system unstable against all disturbances of sufficiently short wavelength. This is unphysical-capillary effects should stabilize a system against short-wavelength perturbations, not destabilize it-and so we conclude that $d_{0}$ must be positive or zero. It is worth noting that for growth-rate curves of the type of Fig. 1(b), $d_{0}>0$ is inconsistent with the statement, sometimes encountered in the literature, that the growth velocity depends only on the difference between the interface temperature and the equilibrium coexistence temperature. For positive curvatures, the equilibrium temperature is lowered by the Gibbs-Thomson effect, so according to this statement the entire curve of intrinsic growth velocity versus interface temperature would then be shifted to the left. This would correctly decrease the growth velocity on the right-hand side of the curve, but would raise it on the left-hand side where the curve slopes upward, thus rendering the system unstable. The more precise statement which is intended is that positive curvature lowers the intrinsic growth velocity (cf. also Refs. 29 and 32).

In the following sections we seek the stability boundary of the steady-state solution. We will look first at two special cases: $k=0$, for which the disturbances preserve the straight-line interface, and which then gives the stability boundary for a one-dimensional system, and $R=0$, for which the laser is absent. We then consider the general case. To locate the stability boundary, we first note that 
on the critical surface for a given $k, \omega(k)$ must be purely imaginary, since $\operatorname{Re}(\omega)$ must be positive on one side of the surface and negative on the other; as noted above, we can have $\omega=0$ only when $R=0$ and $k=0$. We then locate the critical surface by setting

$$
\omega=i \Omega \text {, }
$$

and then writing the radical in the stability equation as $Q+i P$, where $Q$ and $P$ are real and $Q$ satisfies $Q>\left(1+\beta k^{2}\right)^{1 / 2}$. From $(Q+i P)^{2}=1+\beta k^{2}+2 \beta \omega$, we obtain

$$
\Omega=Q P / \beta=Q\left(Q^{2}-1-\beta k^{2}\right)^{1 / 2} / \beta,
$$

which we then use to eliminate $\Omega$ in favor of $Q$ (Coriell and Sekerka ${ }^{51}$ have used a similar method to study oscillatory instabilities in rapid directional solidification of a mixture). This yields two equations-the real and imaginary parts of the stability equation-which we solve for $\beta$. The result is

$\beta=\frac{Q(\alpha-Q)}{(1+R) \alpha+d_{0} k^{2}}=\frac{Q\left(Q^{2}-1\right)}{\left[(1+R) \alpha+\left(1+d_{0}\right) k^{2}\right] Q-\alpha}$.

By cross-multiplying we can obtain a quadratic equation for $Q$, whose solution then yields the critical surface for given $k$. This is useful for a one-dimensional system, whose stability boundary is just the $k=0$ critical surface. However, it is not the best approach for a twodimensional system, for which we use other techniques to locate the stability boundary. Note that for $Q>\alpha$ the middle member of (4.14) is negative and so, since $\beta$ must lie between 0 and 1 , there can be no solution. Since
$Q>\left(1+\beta k^{2}\right)^{1 / 2}$, this implies that no critical curve can have $0<\alpha<1$; thus for $\alpha$ in this range the steady-state solution is linearly stable. Note also that the critical surfaces can go through $\beta=0$ only when $Q=\alpha=1$.

For negative $\alpha$, corresponding to growth on the righthand side of a growth-rate curve of the form of Fig. 1(b), the stability equation (4.11) shows that the steady state is unstable for all $R>0$. To see this, we solve it for $\alpha$, finding

$$
\begin{aligned}
\alpha= & \left(\omega+d_{0} k^{2}\right)\left(1+\beta k^{2}+2 \beta \omega\right)^{1 / 2} \\
& \times\left[\omega+1-(1+R)\left(1+\beta k^{2}+2 \beta \omega\right)^{1 / 2}\right]^{-1} .
\end{aligned}
$$

If we imagine varying $\omega$ with $k=0$, we see that for $R>0, \alpha$ decreases from 0 to $-\infty$ as $\omega$ increases from 0 to the positive zero of the denominator. Turning the argument around, we see that for any negative $\alpha$ the stability equation has a positive real solution $\omega$ corresponding to an unstable mode (with $k=0$ ). This is to be expected on physical grounds, for if the interface moves slightly farther from the laser than its steady-state position, then its temperature drops slightly; if $\alpha$ is negative, this causes the interface to speed up and move even farther ahead of the laser. For self-sustained growth we set $R=0$, and the above argument shows that the steady state is unstable for $\alpha \leq-2 d_{0} / \beta$, which agrees with the results of Temkin and Polyakov. ${ }^{32}$ It is also possible to show from (4.14) that there are no oscillatory instabilities for $\alpha \leq 0$.

\section{ONE-DIMENSIONAL LASER-DRIVEN SYSTEM}

When we set $k=0$, so that we restrict our attention to one-dimensional perturbations, which leave the interface a straight line, the procedure outlined above yields

$$
\beta=\left(\left\{(1+R)^{2} \alpha^{2}+4(1+R) \alpha-\left[1+4(1+R)^{2}\right]\right\}+[(1+R) \alpha-1]\left\{[(1+R) \alpha-3]^{2}+8\left[(1+R)^{2}-1\right]\right\}^{1 / 2}\right)\left[8(1+R)^{3} \alpha\right]^{-1} .
$$

Using (4.13), we then obtain the frequency of the unstable oscillation,

$$
\Omega^{2}=\frac{\alpha^{2}}{2\left(\alpha^{2}-1\right)}\left((1+R)^{2} \alpha^{2}-4(1+R) \alpha+\left[1+2(1+R)^{2}\right]+[(1+R) \alpha-1]\left\{[(1+R) \alpha-3]^{2}+8\left[(1+R)^{2}-1\right]\right\}^{1 / 2}\right) .
$$

Near the stability boundary, the actual interface velocity should have a small oscillatory component whose angular frequency is close to this $\Omega$. Thus in one cycle of the oscillation the interface will have moved a distance of about $\lambda_{\|}=4 \pi D / V_{L} \Omega$ [cf. Eq. (3.7)]. As argued in Sec. III, this should be the wavelength of the surface structure left behind. In fact, a nonlinear analysis ${ }^{52}$ of the motion of the interface when $R$ is small and $\alpha$ and $\beta$ are close to the stability boundary (with $\beta<\frac{2}{3}$ ) confirms this expectation.

When $R$ is large, we find

$$
\beta \approx \frac{\left(\alpha^{2}-4\right)+\alpha\left(\alpha^{2}+8\right)^{1 / 2}}{8 \alpha R}
$$

and

$$
\Omega \approx \alpha R\left[\frac{\left(\alpha^{2}+2\right)+\alpha\left(\alpha^{2}+8\right)^{1 / 2}}{2\left(\alpha^{2}-1\right)}\right]^{1 / 2} .
$$

From this we see that when the steady-state interface "rides" on a steep-sloping laser temperature profile, an instability can only be triggered by small $\beta$, meaning low laser velocity or high heat-loss rate, or by large $\alpha$, meaning a steep growth-velocity curve. Moreover, when the instability does set in, it is with a very high frequency, so that the surface structure it produces has a very short wavelength. This is reasonable, since such a laser profile acts somewhat like a stiff spring, in the sense that small displacements of the interface from its steady-state position result in large changes in its temperature, and hence 
large restoring changes in its velocity. An instability occurs when the fractional change in velocity thus produced is so large-either because $V_{L}$ is small or because $d V^{b} / d T^{b}$ is large-that it overcompensates the perturbation, so that the interface is pushed to the other side of its steady-state position.

In the opposite limit of small $R$ we recover the results of vSW: the stability boundary is given by

$$
\beta \approx\left\{\begin{array}{l}
\frac{\alpha^{2}-1}{4 \alpha}-\frac{(\alpha+1)\left(\alpha^{2}-4 \alpha+1\right)}{4 \alpha(\alpha-3)} R, \quad \alpha>3 \\
\frac{2}{3}+\frac{1}{3} R^{1 / 2}, \quad \alpha=3 \\
\frac{\alpha-1}{\alpha}+\frac{(\alpha-1)(2 \alpha-5)}{\alpha(3-\alpha)} R, \quad \alpha<3
\end{array}\right.
$$

with the imaginary part of the linear growth rate at the boundary given by

$$
\Omega \approx\left\{\begin{array}{l}
\alpha\left[\frac{\alpha-3}{\alpha+1}\right]^{1 / 2}\left[1+\frac{\alpha^{2}-4 \alpha+5}{(\alpha-3)^{2}} R\right], \quad \alpha>3 \\
\frac{3}{2} R^{1 / 4}, \quad \alpha=3 \\
\alpha[2 R /(\alpha-1)(3-\alpha)]^{1 / 2}, \quad \alpha<3
\end{array}\right.
$$

Thus for small gradients of the laser temperature profile, the curve along which the steady-state solution becomes unstable against one-dimensional perturbations moves into previously stable regimes for $\frac{5}{2}<\alpha<2+\sqrt{3}$ or $\frac{3}{5}<\beta<\sqrt{3} / 2$. In this intermediate range of parameters it is then easier to destabilize the interface when it is driven by the laser at small $R$. In addition, the instability which has $\omega$ real when the laser is absent acquires a slow oscillatory component for small $R$. This is because this instability is caused by a balance of stabilizing and destabilizing effects, while the presence of the laser introduces another stabilizing force which tends to overcompensate changes in the interface velocity.

One should bear in mind that the curve defined by (5.1) is not necessarily the stability boundary for a two- dimensional system, because the system could be unstable against finite-wavelength perturbations even if it is stable against those with $k=0$. As we will see in Sec. VII, this does in fact occur for $R$ not too large.

\section{TWO-DIMENSIONAL SELF-SUSTAINED GROWTH}

To examine the possibility of morphological instabilities occurring in self-sustained growth (with no laser present), we set $R=0$ in the stability equation while keeping $k$ arbitrary. vSW investigated the stability of the steady state against $k=0$ perturbations and found that the critical curve at which the system becomes unstable against these perturbations is given by (5.5) with $R=0$. We can find the critical curves for arbitrary $k$ by setting $R=0$ in (4.14) and eliminating $Q$. The stability boundary is given by the envelope of the resulting critical curves, since stability requires that there be no unstable modes.

For large $k,(4.14)$ becomes

$$
\beta \approx \frac{Q(\alpha-Q)}{d_{0} k^{2}} \approx \frac{Q^{2}-1}{\left(1+d_{0}\right) k^{2}},
$$

so that $Q$ will approach a finite value and $\beta \propto 1 / k^{2}$. Thus, large- $k$ instabilities are unimportant since they only set in at very small $\beta$, for which the system is already unstable against $k=0$ perturbations.

We must now investigate the possibility that the system may be stable against perturbations with large $k$ and those with $k=0$, but unstable against those having some intermediate $k$. To check this, we first examine the behavior of the critical curves for small $k$. In this regime, we expect from (4.14) that $Q$ will approach the point where $Q(\alpha-Q) / \alpha$ and $Q(Q+1) / \alpha$ intersect, namely $Q=(\alpha-1) / 2$. This, in fact, is correct provided $\alpha>3$, but is invalid for $\alpha<3$ because the resulting value of $Q$ would be less than 1, while $Q$ must be greater than 1 in order for $\Omega$ to be real. Instead, for small $k$ with $\alpha<3, Q$ approaches 1 , where both the numerator and denominator of the last member of (4.14) are small. An expansion in powers of $k$ then leads to

$$
\beta \approx\left\{\begin{array}{l}
\frac{\alpha+1}{4 \alpha}\left[(\alpha-1)+\frac{(\alpha-1)-d_{0}\left(\alpha^{2}-4 \alpha+1\right)}{\alpha(\alpha-3)} k^{2}\right], \alpha>3 \\
\frac{2}{3}+\left[\frac{1+d_{0}}{27}\right]^{1 / 2}|k|, \alpha=3 \\
\frac{\alpha-1}{\alpha}\left[1+\frac{(\alpha-2)+d_{0}(2 \alpha-5)}{\alpha(3-\alpha)} k^{2}\right], \alpha<3
\end{array}\right.
$$

and

$$
\boldsymbol{\Omega} \approx\left\{\begin{array}{l}
\alpha\left[\frac{\alpha-3}{\alpha+1}\right]^{1 / 2}\left[1+\frac{(\alpha-1)^{2}+2 d_{0}\left(\alpha^{2}-4 \alpha+5\right)}{2 \alpha(\alpha-3)^{2}} k^{2}\right], \alpha>3 \\
{\left[27\left(1+d_{0}\right) / 4\right]^{1 / 4} k^{1 / 2}, \alpha=3} \\
{\left[\alpha\left(\alpha-1+2 d_{0}\right) /(\alpha-1)(3-\alpha)\right]^{1 / 2}|k|, \alpha<3 .}
\end{array}\right.
$$


From this we see that there are, in fact, ranges of the growth parameters $\alpha$ and $\beta$ for which the steady-state solution is stable against $k=0$ perturbations but unstable against those with some small but finite $k$. This occurs where the coefficient of $k^{2}$ in the expansion of $\beta$ in (6.2) is positive: from $\alpha=\left(2+5 d_{0}\right) /\left(1+2 d_{0}\right)$, which lies between 2 and $\frac{5}{2}$, to the zero (with $\alpha>3$ ) of $d_{0} \alpha^{2}-\left(1+4 d_{0}\right) \alpha+\left(1+d_{0}\right)$, which decreases from infinity for $d_{0} \rightarrow 0$ to $2+\sqrt{3}$ as $d_{0} \rightarrow \infty$. Note that the critical curve for $k=0$ reaches the limiting value $\beta=1$ when $\alpha=2+\sqrt{5}$; for this value of $\alpha$ we see that the critical curve for small $k$ lies above that for $k=0$, provided that $d_{0}<(1+\sqrt{5}) / 2=1.61 \ldots$; otherwise it lies below the $k=0$ curve. Thus for $d_{0}$ less than this value, the interface first becomes unstable at some finite $k$ as $\alpha$ is increased with $\beta=1$, while for $d_{0}$ greater than this value, the first instability to set in at $\beta=1$ has $k=0$. Most experiments find interface velocities that are large compared to $(D \Gamma)^{1 / 2}$, and so have $\beta$ near 1 . Thus we see that for sufficiently strong capillarity (which we expect to have in the limit $V_{\mathrm{SS}} \rightarrow \infty, \beta \rightarrow 1$; see Sec. III), the steady state with $\beta \approx 1$ is unstable against $k=0$ perturbations if it is unstable at all; for weaker capillarity, however, it may undergo a morphological instability, being unstable against some finite $k$ but not against the $k=0$ mode.

It is possible to write the exact stability boundary of the model parametrically, using $Q$ as a parameter. This analysis and its result are presented in Appendix B for general $R$. It is much simpler and more illustrative to look at the case $d_{0}=0$, which is qualitatively similar to the general case [for $d_{0}<(1+\sqrt{5}) / 2$ ], and for which the characteristics of the stability boundary can be written explicitly. For $R=0$, the critical curve for a fixed $k$ is found by solving

$$
\beta=\frac{Q(\alpha-Q)}{\alpha}=\frac{Q\left(Q^{2}-1\right)}{\left(\alpha+k^{2}\right) Q-\alpha} .
$$

The stability boundary occurs where the critical $\beta$ for fixed $\alpha$ has a maximum as a function of $k$. This is found by setting

$$
\begin{aligned}
0=\left[\frac{\partial \beta}{\partial k^{2}}\right]_{\alpha}=\frac{\alpha-2 Q}{\alpha} Q^{\prime}= & \frac{3 Q^{2}-1}{\left(\alpha+k^{2}\right) Q-\alpha} Q^{\prime} \\
& -\frac{Q\left(Q^{2}-1\right)\left[\left(\alpha+k^{2}\right) Q^{\prime}+Q\right]}{\left[\left(\alpha+k^{2}\right) Q-\alpha\right]^{2}}
\end{aligned}
$$

where $Q^{\prime}$ denotes $\left(\partial Q / \partial k^{2}\right)_{\alpha, \beta}$. From the middle member of this equation we see that this maximum is attained for $Q=\alpha / 2$ (the alternative $Q^{\prime}=0$ leads to the uninteresting results $Q=1, \beta=0$, and $\alpha=1$ ). Substituting this into the middle member of $(6.4)$ we find

$$
\beta=\alpha / 4 \text {, }
$$

and the right member then gives

$$
k^{2}=2(\alpha-2) / \alpha
$$

for the wave number of the perturbation against which the system is unstable at the stability boundary. We then find, using (4.13), that the imaginary growth rate of this perturbation is

$$
\Omega=\sqrt{\alpha(\alpha-2)} .
$$

If, near the onset of this instability, the interface restabilizes into a sinusoidal wave of dimensionless wave number $k$ propagating with a velocity which has an oscillatory component of dimensionless angular frequency $\Omega$, then the surface pattern it leaves behind will have an aspect ratio

$$
\frac{\lambda_{\|}}{\lambda_{\perp}}=\frac{4 \pi D}{V_{L} \Omega} / \frac{4 \pi D}{V_{L} k}=\frac{k}{\Omega}=\frac{\sqrt{2}}{\alpha}
$$

which varies from $\sqrt{2} / 2$ to $\sqrt{2} / 4$ along the part of the stability boundary $\left(2<\alpha<4\right.$ or $\left.\frac{1}{2}<\beta<1\right)$ for which morphological instabilities occur.

\section{TWO-DIMENSIONAL LASER-DRIVEN GROWTH}

In the general case of nonzero $R$ and $k$, the stability boundary can also be located parametrically as in the special case $R=0$. The details of the procedure are presented in Appendix B. Again, the results are much easier to appreciate in the simple case $d_{0}=0$, although, as we will see below, one qualitative feature of the stability boundary for general $d_{0}>0$ is missing in this special case. For this case, the stability boundary can again be found as it was in the preceding section for $R=0$. It is given explicitly by

$$
\beta=\alpha / 4(1+R) .
$$

The wave number of the perturbation against which the steady state first becomes unstable as we approach this stability boundary is

$$
k^{2}=2-4(1+R) / \alpha,
$$

from which we see that the finite- $k$ part of the stability boundary meets the section at which $k=0$ instabilities occur first at $\alpha=2(1+R)$ and $\beta=\frac{1}{2}$. For smaller $\alpha$ or $\beta$ the system first becomes unstable against one-dimensional $(k=0)$ perturbations. Note that the transverse wavelength $\lambda_{\perp}=4 \pi D / V_{L} k$ for the unstable perturbation increases with $R$. The frequency of the unstable oscillations at the stability boundary is given by

$$
\Omega=\sqrt{(1+R) \alpha[(1+R) \alpha-2]},
$$

which remains finite even at $\alpha=2(1+R)$, provided $R$ is positive. This reflects the fact that for $R>0$, even the $k=0$ instabilities are oscillatory. The aspect ratio of the pattern that this instability would leave behind is

$$
\begin{aligned}
\frac{\lambda_{\|}}{\lambda_{\perp}}=\frac{k}{\Omega} & =\frac{1}{\alpha}\left[\frac{2[\alpha-2(1+R)]}{(1+R)[(1+R) \alpha-2]}\right]^{1 / 2} \\
& =\frac{1}{2(1+R) \beta}\left[\frac{\beta-1 / 2}{2(1+R)^{2} \beta-1}\right]^{1 / 2},
\end{aligned}
$$

which is a decreasing function of $R$ for fixed $\alpha$ or fixed $\beta$. We see, then, from (7.1), that if the interface "rides" the 
laser temperature profile $T_{L}(x)$ at a location where its slope $R$ is large, then it is difficult to make it unstable. This is because small excursions of the interface position $x^{b}$ ahead of its steady-state value give rise to an appreciable decrease in $T_{L}\left(x^{b}\right)$, which tends to slow the interface down and so return it to its steady-state position. When the interface does become unstable, the frequency of the unstable oscillations, given by (7.3), is higher than when $R$ is smaller, so that $\lambda_{\|}$decreases with increasing $R$, while $\lambda_{\perp}$ increases.

For nonzero $d_{0}$, a new effect arises-the region of morphological instabilities shrinks as the slope $R$ of the laser temperature profile increases. This is found by examining expression (B10) derived in Appendix B for the wave number of the unstable mode at the stability boundary. The zeros of this expression mark points on the stability boundary at which the instability crosses over from occurring at $k=0$ to occurring at some finite $k$. As pointed out above, for $R=0$ there will be one such point at $\alpha=\left(2+5 d_{0}\right) /\left(1+2 d_{0}\right), \beta=\left(1+3 d_{0}\right) /\left(2+5 d_{0}\right)$, and if $d_{0}>(1+\sqrt{5}) / 2$ there will be another where $\alpha$ is the zero (with $\alpha>3$ ) of $d_{0} \alpha^{2}-\left(1+4 d_{0}\right) \alpha+\left(1+d_{0}\right)$. As $R$ is increased, these values change. If $d_{0}$ is less than $(1+\sqrt{5}) / 2$, then for $R=0$ there is only one of these crossover points, but another appears at $\beta=1$ when $R$ reaches a value given by

$$
(1+R)^{2}=\frac{\left(1+11 d_{0}+33 d_{0}^{2}+31 d_{0}^{3}\right)+\left(1+6 d_{0}+7 d_{0}^{2}\right)\left(1+10 d_{0}+17 d_{0}^{2}\right)^{1 / 2}}{16 d_{0}\left(1+2 d_{0}\right)^{2}}
$$

This value of $R$ grows as $\left(8 d_{0}\right)^{-1 / 2}$ as $d_{0} \rightarrow 0$, and is equal to zero for $d_{0}=(1+\sqrt{5}) / 2$. As $R$ is increased further, the region of morphological instabilities disappears completely when $R$ satisfies

$$
\begin{aligned}
(1+R)^{2}=\left[16 d_{0}\left(1+2 d_{0}\right)^{3}\right]^{-1}\{ & \left(1+10 d_{0}-3 d_{0}^{2}-140 d_{0}^{3}-236 d_{0}^{4}\right) \\
& \left.+\left(1+5 d_{0}+22 d_{0}^{2}\right)\left[\left(1+2 d_{0}\right)\left(1+3 d_{0}\right)\left(1+5 d_{0}+22 d_{0}^{2}\right)\right]^{1 / 2}\right\} .
\end{aligned}
$$

When $R$ exceeds this value [which diverges as $\left(8 d_{0}\right)^{-1 / 2}$ as $d_{0} \rightarrow 0$, decreases to 0.0475 as $d_{0} \rightarrow \infty$, and is already as small as 0.1 for $d_{0} \approx \frac{1}{2}$ ], there is no region of morphological instability - the first instability to occur at the stability boundary has $k=0$. Thus morphological instabilities, which cannot be suppressed even by infinite $d_{0}$ when the laser is absent, can be suppressed by making $R$ large enough as long as $d_{0}$ is greater than zero.

\section{APPENDIX A}

To derive the stability equation (4.11), we start with the steady-state solution (4.5) of the diffusion equation (4.1) with the interface-velocity condition (4.3). We add an infinitesimal perturbation to this steady-state solution,

$$
\begin{aligned}
& x^{b}(y, t)=x_{\mathrm{SS}}^{b}+\epsilon \exp \left[i \frac{V_{L}}{2 D} k y+\frac{V_{L}^{2}}{2 D} \omega t\right], \\
& T(x, y, t)=T_{\mathrm{SS}}(x)+\epsilon \hat{T}(x) \exp \left[i \frac{V_{L}}{2 D} k y+\frac{V_{L}^{2}}{2 D} \omega t\right],
\end{aligned}
$$

and insert this into the basic equations, keeping only terms of first order in $\epsilon$. The interface-velocity condition (4.3) gives

$$
\frac{V_{L}^{2}}{2 D} \omega=\left.\frac{\partial V^{b}}{\partial T^{b}}\right|_{T_{\mathrm{SS}}^{b}} \hat{T}\left(x_{\mathrm{SS}}^{b}\right)+\left.\frac{\partial V^{b}}{\partial \kappa}\right|_{0} \frac{V_{L}^{2}}{4 D^{2}} k^{2},
$$

or

$$
\omega+d_{0} k^{2}=\frac{2 D \alpha}{q V_{L} \sqrt{\beta}} \hat{T}\left(x_{\mathrm{SS}}^{b}\right)
$$

To find the correction $\widehat{T}(x)$ to the temperature field, it is convenient to change variables in the diffusion equation from $x$ to

$$
z=x-x^{b}(y, t) .
$$

The resulting equation reads

$$
\begin{aligned}
\frac{\partial T}{\partial t}= & D\left\{\left[1+\left[\frac{\partial x^{b}}{\partial y}\right]^{2}\right] \frac{\partial^{2} T}{\partial z^{2}}-2 \frac{\partial x^{b}}{\partial y} \frac{\partial^{2} T}{\partial z d y}+\frac{\partial^{2} T}{\partial y^{2}}\right\} \\
& +\left[V_{L}-D \frac{\partial^{2} x^{b}}{\partial y^{2}}+\frac{\partial x^{b}}{\partial t}\right] \frac{\partial T}{\partial z} \\
& -\Gamma\left(T-T^{0}\right)+p\left(z+x^{b}\right)+q V^{b} \delta(z),
\end{aligned}
$$

where

$$
p(x)=J(x) / c d .
$$

The advantage of this choice of variables is that the interface is always at $z=0$, and so the $\delta$ function in the equation is always localized at a known value of $z$, no matter what $x^{b}(y, t)$ does. In order to derive the stability equation from its nascent form (A3), we need the value of $\widehat{T}$ at $z=0$. Substituting (A1) into (A5), linearizing in $\epsilon$, and rearranging yields

$$
\begin{aligned}
0= & D \frac{d^{2} \widehat{T}}{d z^{2}}+V_{L} \frac{d \widehat{T}}{d z}-\left[\Gamma+\frac{V_{L}^{2}}{4 D}\left(k^{2}+2 \omega\right)\right] \widehat{T} \\
& +\frac{V_{L}^{2}}{4 D}\left(k^{2}+2 \omega\right) \frac{d T_{\mathrm{SS}}}{d z}+\frac{d p\left(z+x_{\mathrm{SS}}^{b}\right)}{d z}+\frac{q V_{L}^{2}}{2 D} \omega \delta(z)
\end{aligned}
$$

The Green's function $G_{\mathrm{SS}}^{k, \omega}$ for this equation is the same as $G_{\text {SS }}$ given in (4.6), except that $\Gamma$ is replaced by the factor in large parentheses in (A7), 


$$
\begin{aligned}
G_{\mathrm{SS}}^{k, \omega}(z) & =\left[V_{L}^{2}\left(1+k^{2}+2 \omega\right)+4 D \Gamma\right]^{-1 / 2} \exp \left(-\frac{V_{L}}{2 D} z-\frac{\left[V_{L}^{2}\left(1+k^{2}+2 \omega\right)+4 D \Gamma\right]^{1 / 2}}{2 D}|z|\right) \\
& =\frac{1}{V_{L}}\left(\frac{\beta}{1+\beta k^{2}+2 \beta \omega}\right]^{1 / 2} \exp \left\{-\frac{V_{L}}{2 D}\left[z+\left(\frac{1+\beta k^{2}+2 \beta \omega}{\beta}\right)^{1 / 2}|z|\right]\right\} .
\end{aligned}
$$

Thus $\hat{T}$ is given by

$$
\begin{aligned}
\widehat{T}(z) & =\frac{q V_{L}^{2}}{2 D} \omega G_{\mathrm{SS}}^{k, \omega}(z)+\int_{-\infty}^{\infty} G_{\mathrm{SS}}^{k, \omega}\left(z-z^{\prime}\right) \frac{d}{d z^{\prime}}\left[\frac{V_{L}^{2}}{4 D}\left(k^{2}+2 \omega\right) T_{\mathrm{SS}}\left(z^{\prime}\right)+p\left(z^{\prime}+x_{\mathrm{SS}}^{b}\right)\right] d z^{\prime} \\
& =\frac{q V_{L}^{2}}{2 D} \omega G_{\mathrm{SS}}^{k, \omega}(z)+\frac{d}{d z} \int_{-\infty}^{\infty} G_{\mathrm{SS}}^{k, \omega}\left(z-z^{\prime}\right)\left[\frac{V_{L}^{2}}{4 D}\left(k^{2}+2 \omega\right) T_{\mathrm{SS}}\left(z^{\prime}\right)+p\left(z^{\prime}+x_{\mathrm{SS}}^{b}\right)\right] d z^{\prime} .
\end{aligned}
$$

From (4.5) we see that $T_{\mathrm{SS}}$ satisfies

$$
T_{\mathrm{SS}}\left(z^{\prime}\right)=T^{0}+\int_{-\infty}^{\infty} G_{\mathrm{SS}}\left(z^{\prime}-z^{\prime \prime}\right)\left[p\left(z^{\prime \prime}+x_{\mathrm{SS}}^{b}\right)+q V_{L} \delta\left(z^{\prime \prime}\right)\right] d z^{\prime \prime},
$$

so that the term in (A9) involving $T_{\mathrm{SS}}$ has a convolution of the two Green's functions. However, it is easy to see (using Fourier transforms, for instance) that this convolution is

$$
\frac{V_{L}^{2}}{2 D}\left(k^{2}+2 \omega\right) \int_{-\infty}^{\infty} G_{\mathrm{SS}}^{k, \omega}\left(z-z^{\prime}\right) G_{\mathrm{SS}}\left(z^{\prime}-z^{\prime \prime}\right) d z^{\prime}=G_{\mathrm{SS}}\left(z-z^{\prime \prime}\right)-G_{\mathrm{SS}}^{k, \omega}\left(z-z^{\prime \prime}\right),
$$

and so

$$
\begin{aligned}
\widehat{T}(z) & =\frac{q V_{L}^{2}}{2 D} \omega G_{\mathrm{SS}}^{k, \omega}(z)+\frac{d}{d z} \int_{-\infty}^{\infty}\left\{\left[G_{\mathrm{SS}}\left(z-z^{\prime}\right)-G_{\mathrm{SS}}^{k, \omega}\left(z-z^{\prime}\right)\right]\left[p\left(z^{\prime}+x_{\mathrm{SS}}^{b}\right)+q V_{L} \delta\left(z^{\prime}\right)\right]+G_{\mathrm{SS}}^{k, \omega}\left(z-z^{\prime}\right) p\left(z^{\prime}+x_{\mathrm{SS}}^{b}\right)\right\} \\
& =\frac{q V_{L}^{2}}{2 D} \omega G_{\mathrm{SS}}^{k, \omega}(z)+q V_{L} \frac{d}{d z}\left[G_{\mathrm{SS}}(z)-G_{\mathrm{SS}}^{k, \omega}(z)\right]+\frac{d T_{L}}{d z} .
\end{aligned}
$$

Evaluating this explicitly at $z=0$ gives

$$
\hat{T}\left(x=x_{\mathrm{SS}}^{b}\right)=\frac{V_{L}}{2 D} q \sqrt{\beta}\left(\frac{\omega+1}{\left(1+\beta \kappa^{2}+2 \beta \omega\right)^{1 / 2}}-1\right)+\left.\frac{d T_{L}}{d x}\right|_{x_{\mathrm{SS}}^{b}}=\frac{V_{L}}{2 D} q \sqrt{\beta}\left(\frac{\omega+1}{\left(1+\beta k^{2}+2 \beta \omega\right)^{1 / 2}}-(1-R)\right) .
$$

Substituting this into (A3) and rearranging gives the stability equation

$$
\left[\omega+(1+R) \alpha+d_{0} k^{2}\right]\left(1+\beta k^{2}+2 \beta \omega\right)^{1 / 2}=\alpha(\omega+1) .
$$

\section{APPENDIX B}

In order to locate the stability boundary of our model, we start with equation (4.14) which gives the critical surface for a given $k$,

$\beta=\frac{Q(\alpha-Q)}{(1+R) \alpha+d_{0} k^{2}}=\frac{Q\left(Q^{2}-1\right)}{\left[(1+R) \alpha+\left(1+d_{0}\right) k^{2}\right] Q-\alpha}$.

The parameter $Q$ is the real part of $\left(1+\beta k^{2}+2 i \beta \Omega\right)^{1 / 2}$, where the linear growth rate of a perturbation of wave number $k$ is $\omega=i \Omega$, which is purely imaginary at the critical surface. At the stability boundary, only the perturbation with a certain wave number will have $\operatorname{Re}(\omega)=0$; the system will be stable against all others, so their linear growth rates will have negative real parts. This can occur in two ways: either $\operatorname{Re}(\omega)$ vanishes at $k=0$ and is negative for all nonzero $k$, or it has a maximum value of zero for some nonzero $k$ and is negative for all other $k$. In general, the stability boundary will consist of portions of both types-one on which the instability has $k=0$ and one on which a morphological instability, with nonzero $k$, is occurring.

To find the $k=0$ portion of the stability boundary we need only find the critical surface for $k=0$, for which (B1) reduces to

$$
\beta=\frac{Q(\alpha-Q)}{(1+R) \alpha}=\frac{Q\left(Q^{2}-1\right)}{[(1+R) Q-1] \alpha} .
$$

From this we obtain parametric equations for $\alpha$ and $\beta$, using $Q$ and $R$ as parameters. Solving the last two members of (B2) for $\alpha$, we obtain

$$
\alpha=\frac{(1+R)\left(2 Q^{2}-1\right)-Q}{(1+R) Q-1},
$$

and substituting this back into (B2) gives

$$
\beta=\frac{Q\left(Q^{2}-1\right)}{(1+R)\left(2 Q^{2}-1\right)-Q} .
$$

The imaginary growth rate of the perturbation at the critical surface is found from (4.13),

$$
\Omega=\frac{(1+R)\left(2 Q^{2}-1\right)-Q}{\left(Q^{2}-1\right)^{1 / 2}} .
$$

From this we see that the parameter $Q$ must be at least as 
large as 1 , otherwise $\Omega$ would not be real. At $Q=1$ we have $\alpha=1$ and $\beta=0$, except when $R=0$, for which we have $\alpha=3$ and $\beta=\frac{2}{3}$ when $Q=1$. This occurs because in the case of self-sustained growth, $R=0$, the critical curve for $k=0$ includes the part of the curve $\beta=(\alpha-1) / \alpha$ having $\alpha<3$, at which the instability is not oscillatory but has $\omega$ real.

To find the finite- $k$, morphological-instability part of the stability boundary, we look at the boundary as the envelope of the critical surfaces in $\alpha-\beta-R$ space. If we adopt this viewpoint, it becomes clear that any point on the stability boundary which is on a critical curve for nonzero $k$ may be located by extremizing $\beta$, say, as a function of $k^{2}$ subject to (B1) with $\alpha$ and $R$ fixed. This will fail, of course, if the $\beta$ direction is tangent to the stability boundary, but we will find that this does not happen in our model. Differentiating the first and middle members of (B1) with respect to $k^{2}$ gives

$$
\left[\frac{\partial \beta}{\partial k^{2}}\right]_{\alpha}=0=\frac{\alpha-2 Q}{(1+R) \alpha+d_{0} k^{2}} Q^{\prime}-\frac{d_{0} Q(\alpha-Q)}{\left[(1+R) \alpha+d_{0} k^{2}\right]^{2}},
$$

which we can solve for $Q^{\prime}=\partial Q / \partial k^{2}$. Differentiating the last member of (B1), substituting for $Q^{\prime}$, and solving for $k^{2}$ gives

$$
k^{2}=\frac{(1+R) \alpha Q\left[\left(Q^{2}-1\right)(\alpha-2 Q)-d_{0} \alpha\left(Q^{2}+1\right)+2 Q d_{0}\right]+d_{0} \alpha\left(3 Q^{2}-1\right)(\alpha-Q)}{d_{0}\left(1+d_{0}\right) Q\left[\left(Q^{2}+1\right) \alpha-2 Q\right]}
$$

We now use this to eliminate $k^{2}$ in (B1) and obtain a parametric equation for $\alpha$,

$$
\alpha=\frac{2(1+R) Q^{3}+d_{0} Q^{2}[4(1+R) Q+3]+d_{0}^{2}\left(6 Q^{2}-1\right)}{\left(1+d_{0}\right) Q\left[(1+R) Q+2 d_{0}\right]}
$$

Substituting this and (B7) into (B1) yields the equation for $\beta$,

$$
\beta=\frac{\left(1+d_{0}\right) Q^{2}\left[Q^{2}+d_{0}\left(2 Q^{2}+1\right)\right]}{2(1+R) Q^{3}+d_{0} Q^{2}[4(1+R) Q+3]+d_{0}^{2}\left(6 Q^{2}-1\right)} .
$$

We find the wave number of the unstable perturbation at the stability boundary by substituting (B8) into (B7), which gives

$$
\begin{aligned}
k^{2}= & \left\{Q[Q-(1+R)]+d_{0}\left[\left(4 Q^{2}-1\right)-(1+R) Q\left(2 Q^{2}+1\right)\right]\right\}\left\{2(1+R) Q^{3}+d_{0} Q^{2}[4(1+R) Q+3]+d_{0}^{2}\left(6 Q^{2}-1\right)\right\} \\
& \times\left\{\left(1+d_{0}\right)^{2} Q^{2}\left[(1+R) Q+2 d_{0}\right]\left[Q^{2}+d_{0}\left(2 Q^{2}+1\right)\right]\right\}^{-1} .
\end{aligned}
$$

Finally, the imaginary growth rate of the perturbation is obtained from (4.13),

$$
\begin{aligned}
\Omega^{2}= & \left\{Q^{2}[(1+R) Q-1]+d_{0}\left[3(1+R) Q^{3}-\left(2 Q^{2}+1\right)\right]\right\}\left\{2(1+R) Q^{3}+d_{0} Q^{2}[4(1+R) Q+3]+d_{0}^{2}\left(6 Q^{2}-1\right)\right\}^{2} \\
& \times\left\{\left(1+d_{0}\right)^{3} Q^{2}\left[(1+R) Q+2 d_{0}\right]\left[Q^{2}+d_{0}\left(2 Q^{2}+1\right)\right]^{2}\right\}^{-1} .
\end{aligned}
$$

In order for these expressions to give us part of the stability boundary, the values of $k^{2}$ and $\Omega^{2}$ coming from (B10) and (B11) must be positive. Expression (B11) is in fact positive for all $Q>1$ and $R>0$, but (B10) vanishes when $Q$ and $R$ satisfy

$$
1+R=\left[\left(1+4 d_{0}\right) Q^{2}-d_{0}\right] / Q\left[2 d_{0} Q^{2}+\left(1+d_{0}\right)\right] .
$$

This function is plotted in Fig. 8. It passes through $R=0$ when $Q=1$ and when

$$
Q=Q_{u}=\left[\left(1+2 d_{0}\right)+\left(1+4 d_{0}+12 d_{0}^{2}\right)^{1 / 2}\right] / 4 d_{0},
$$

and has a maximum at

$$
Q^{2}=Q_{m}^{2}=\frac{\left(1+5 d_{0}+10 d_{0}^{2}\right)+\left[\left(1+2 d_{0}\right)\left(1+3 d_{0}\right)\left(1+5 d_{0}+22 d_{0}^{2}\right)\right]^{1 / 2}}{4 d_{0}\left(1+4 d_{0}\right)},
$$

whose height is given by

$$
\begin{aligned}
(1+R)^{2}=\left[16 d_{0}\left(1+d_{0}\right)^{3}\right]^{-1}\{ & \left(1+10 d_{0}-3 d_{0}^{2}-140 d_{0}^{3}-236 d_{0}^{4}\right) \\
& \left.+\left(1+5 d_{0}+22 d_{0}^{2}\right)\left[\left(1+2 d_{0}\right)\left(1+3 d_{0}\right)\left(1+5 d_{0}+22 d_{0}^{2}\right)\right]^{1 / 2}\right\} .
\end{aligned}
$$

If $R$ lies above the curve, then the value of $k^{2}$ obtained from (B10) will be negative. Thus the curve in $\alpha$ - $\beta$ - $R$ space on which the parameters $Q$ and $R$ satisfy (B12) marks the place where the zero- $k$ and finite- $k$ portions of the stability 
boundary meet. The morphological instability is suppressed entirely for $R$ greater than the maximum value given by (B15). The point on the curve (B12) corresponding to $\beta=1$ is given by

$$
Q^{2}=Q_{1}^{2}=\left[\left(1+5 d_{0}\right)+\left(1+10 d_{0}+17 d_{0}^{2}\right)^{1 / 2}\right] / 4 d_{0} \text {. }
$$

This is always greater than the value $Q_{m}$ of $Q$ at the maximum, so that for $R$ less than its value at the maximum, morphological instabilities do indeed occur for some $\beta$ less than 1 . For $d_{0}>(1+\sqrt{5}) / 2$, we have $Q_{1}$ greater than $Q_{u}$, so that for all $R$ the stability boundary has $k=0$ both for small $\beta$ and for $\beta$ near 1, with morphological instabilities only occurring at intermediate $\beta$ (and $R$ less than the maximum). For smaller $d_{0}$ the opposite situation obtains: $Q_{1}$ is less than $Q_{u}$, and so for $R$ less than the value given by (B12) with $Q=Q_{1}$, which is given by

$$
(1+R)^{2}=\frac{\left(1+11 d_{0}+33 d_{0}^{2}+31 d_{0}^{3}\right)+\left(1+6 d_{0}+7 d_{0}^{2}\right)\left(1+10 d_{0}+17 d_{0}^{2}\right)^{1 / 2}}{16 d_{0}\left(1+2 d_{0}\right)^{2}}
$$

the region of morphological instabilities on the stability boundary extends all the way to $\beta=1$.

In identifying the results of the above calculations as the $k=0$ and $k \neq 0$ parts of the stability boundary, we are tacitly assuming that (i) if $\operatorname{Re}(\omega)$, as a function of $k$ with $\alpha, \beta$, and $R$ fixed, has a maximum of zero at some nonzero $k$, then it is negative for all other $k$, and (ii) if $(\alpha, \beta, R)$ lies on the supposed $k=0$ part of the stability boundary, then at that point $\operatorname{Re}(\omega)$, which vanishes at $k=0$, is negative for all nonzero $k$. Both of these statements can be shown to be true. To establish (i), assume that $(\alpha, \beta, R)$ is given by (B8) and (B9) with $Q$ and $R$ being such that $k^{2}$, given by (B10), is positive. For (i) to fail, there must be another value of $k$ at which $\operatorname{Re}(\omega)$ vanishes. There would also be some value of the parameter, call it $\widetilde{Q} \neq Q$, at which (B1) is satisfied for this $k$ :

$\beta=\frac{\widetilde{Q}(\alpha-\widetilde{Q})}{(1+R) \alpha+d_{0} k^{2}}=\frac{\widetilde{Q}\left(\widetilde{Q}^{2}-1\right)}{\left[(1+R) \alpha+\left(1+d_{0}\right) k^{2}\right] \widetilde{Q}-\alpha}$.

From the first two members we find that $k^{2}$ would be given by

$$
k^{2}=\frac{\widetilde{Q}(\alpha-\widetilde{Q})}{d_{0} \beta}-\frac{(1+R) \alpha}{\beta},
$$

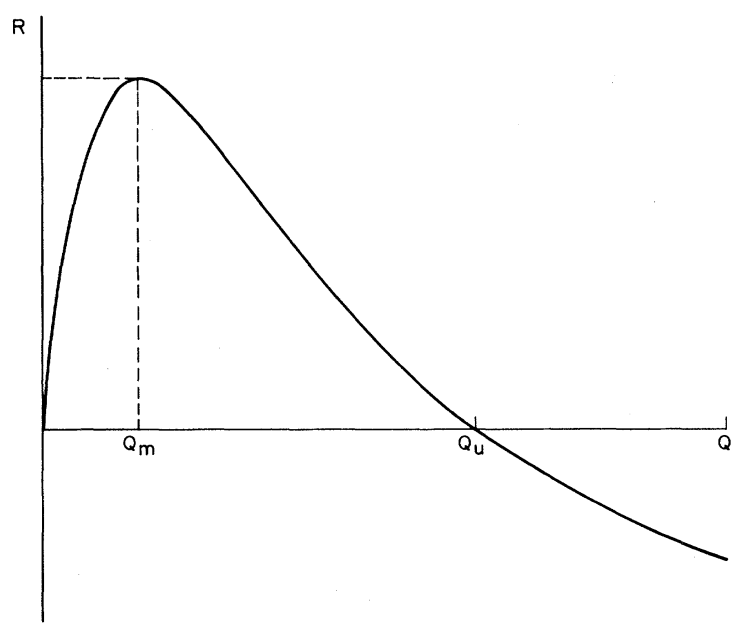

FIG. 8. Plot of $R(Q)$ defined by (B12). For $Q$ and $R$ below the curve, morphological instabilities occur for smaller $\alpha$ than $k=0$ instabilities. and, substituting this back into the last member of (B18), we find that $\widetilde{Q}$ would satisfy the cubic equation

$$
\begin{aligned}
\left(1+2 d_{0}\right) \widetilde{Q}^{3}-\left(1+d_{0}\right) \alpha \widetilde{Q}^{2} & \\
+ & {\left[(1+R) \alpha \beta-d_{0}\right] \widetilde{Q}+d_{0} \alpha \beta=0 . }
\end{aligned}
$$

If we now substitute (B8) and (B9) for $\alpha$ and $\beta$ into this equation, it reduces to

$$
\begin{array}{r}
(\widetilde{Q}-Q)^{2}\left\{\left(1+2 d_{0}\right) Q\left[(1+R) Q+2 d_{0}\right] \widetilde{Q}\right. \\
\left.+d_{0}\left[Q^{2}+d_{0}\left(2 Q^{2}+1\right)\right]\right\}=0 .
\end{array}
$$

Since $Q$ must be at least as large as 1 , the only allowable solution of this equation is $\widetilde{Q}=Q$. Thus there is no other $k$ at which $\operatorname{Re}(\omega)$ vanishes. To prove (ii) we now assume that $(\alpha, \beta, R)$ lies on the calculated $k=0$ part of the stability boundary. That is, $\alpha$ and $\beta$ are given by (B3) and (B4) with $Q$ and $R$ such that $k^{2}$ given by (B10) would be negative, i.e., $R$ is greater than the value given by (B12). Again, for (ii) to fail there would be some nonzero $k$ and a related $\widetilde{Q} \neq Q$ at which $\operatorname{Re}(\omega)$ would vanish, and $k$ and $\widetilde{Q}$ would satisfy (B19) and (B20). Since $k$ must be real, $k^{2}$ given by (B19) must be positive; upon substituting (B3) and (B4) into (B19) we find that this restricts $\widetilde{Q}$ to lie between $Q$ and $(1+R)\left(Q^{2}-1\right) /[(1+R) Q-1]$. Substituting (B3) and (B4) into (B20) shows that $\widetilde{Q}$ would satisfy

$$
\begin{aligned}
(\widetilde{Q}-Q) & \left\{\left(1+2 d_{0}\right)[(1+R) Q-1] \widetilde{Q}^{2}\right. \\
- & {\left[(1+R)\left(Q^{2}-1-d_{0}\right)+d_{0} Q\right] \widetilde{Q} } \\
- & \left.d_{0}\left(Q^{2}-1\right)\right\}=0 .
\end{aligned}
$$

Note that the quantity in braces has one positive and one negative zero, so that if the positive zero were to lie between the limits given above, the quantity would then be positive at one limit and negative at the other. At

$$
\widetilde{Q}=(1+R)\left(Q^{2}-1\right) /[(1+R) Q-1],
$$

it is easily seen to be positive; at $\widetilde{Q}=Q$ it is equal to

$$
(1+R) Q\left[2 d_{0} Q^{2}+\left(1+d_{0}\right)\right]-\left[\left(1+4 d_{0}\right) Q^{2}-d_{0}\right],
$$

which is also positive if, as we have assumed, $R$ is greater than the value given by (B12). Thus our identification of the stability boundary is, in fact, correct.

We may specialize the above results to the case of self- 
sustained crystallization by setting $R=0$ everywhere. The stability boundary is given parametrically by

$$
\alpha=\frac{2 Q^{3}+d_{0} Q^{2}(4 Q+3)+d_{0}^{2}\left(6 Q^{2}-1\right)}{\left(1+d_{0}\right) Q\left(Q+2 d_{0}\right)},
$$

$$
\beta=\frac{\left(1+d_{0}\right) Q^{2}\left[Q^{2}+d_{0}\left(2 Q^{2}+1\right)\right]}{2 Q^{3}+d_{0} Q^{2}(4 Q+3)+d_{0}^{2}\left(6 Q^{2}-1\right)} .
$$

The wave number and imaginary growth rate of the unstable mode at the boundary satisfy

$$
\begin{aligned}
& k^{2}=\frac{(Q-1)\left[Q-d_{0}\left(2 Q^{2}-2 Q-1\right)\right]\left[2 Q^{3}+d_{0} Q^{2}(4 Q+3)+d_{0}^{2}\left(6 Q^{2}-1\right)\right]}{\left(1+d_{0}\right)^{2} Q^{2}\left(Q+2 d_{0}\right)\left[Q^{2}+d_{0}\left(2 Q^{2}+1\right)\right]} \\
& \Omega^{2}=(Q-1)\left[Q^{2}+d_{0}\left(3 Q^{2}+Q+1\right)+2 d_{0}^{2}(Q+1)\right] \frac{\left[2 Q^{3}+d_{0} Q^{2}(4 Q+3)+d_{0}^{2}\left(6 Q^{2}-1\right)\right]^{2}}{\left(1+d_{0}\right)^{3} Q^{2}\left(Q+2 d_{0}\right)\left[Q^{2}+d_{0}\left(2 Q^{2}+1\right)\right]^{2}}
\end{aligned}
$$

As we can see from these equations, the smallest allowable value of $Q$ is 1 . At $Q=1,(\mathrm{~B} 23)$ and (B24) give

$\alpha=\left(2+5 d_{0}\right) /\left(1+2 d_{0}\right), \quad \beta=\left(1+3 d_{0}\right) /\left(2+5 d_{0}\right)$

which is the point at which the finite- $k$ part of the stability boundary meets the curve $\beta=(\alpha-1) / \alpha$, on which the system becomes unstable against the $k=0$ mode with a real growth rate, as discussed in Sec. III. As $\beta$ increases from zero, the stability boundary follows this curve to the point (B27), from which it then follows the morphological-instability curve given by (B23) and (B24). For $d_{0}<(1+\sqrt{5}) / 2$, it follows this curve until it reaches the limiting value $\beta=1$. For $d_{0}>(1+\sqrt{5}) / 2$, it follows the curve until $Q$ reaches $Q_{u}$, at which point it joins the curve of oscillatory instabilities with $k=0$, which is given by $\beta=\left(\alpha^{2}-1\right) / 4 \alpha$.
*Present and permanent address.

${ }^{1}$ G. Gore, Philos. Mag. 9, 73 (1855).

${ }^{2}$ C. C. Coffin and S. Johnston, Proc. R. Soc. London, Ser. A 146, 564 (1934).

${ }^{3}$ W. Lotmar, Helv. Phys. Acta 18, 369 (1945).

${ }^{4}$ A. Götzberger, Z. Phys. 142, 182 (1955).

${ }^{5}$ F. M. Aymerich and A. Delunas, Phys. Status Solidi A 31, 165 (1975).

${ }^{6}$ O. Bostanjoglo and G. Schlotzhauer, Phys. Status Solidi A 68, 555 (1981).

${ }^{7}$ C. E. Wickersham, G. Bajor, and J. E. Green, Solid State Commun. 27, 17 (1978).

${ }^{8}$ C. E. Wickersham, Ph.D. thesis, University of Illinois at Urbana-Champaign (1978)

${ }^{9}$ C. E. Wickersham, G. Bajor, and J. E. Greene (unpublished).

${ }^{10}$ T. Takamori, R. Messier, and R. Roy, Appl. Phys. Lett. 20, 201 (1972); J. Mater. Sci. 9, 159 (1974).

${ }^{11}$ R. Messier, T. Takamori, and R. Roy, Solid State Commun. 16, 311 (1975).

${ }^{12}$ A. Mineo, A. Matsuda, T. Kurosu, and M. Kikuchi, Solid State Commun. 13, 329 (1973).

${ }^{13}$ A. Matsuda, A. Mineo, T. Kurosu, and M. Kikuchi, Solid State Commun. 13, 1165 (1973).

${ }^{14}$ M. Kikuchi, A. Matsuda, T. Kurosu, A. Mineo, and K. J. Callanan, Solid State Commun. 14, 713 (1974).

${ }^{15}$ K. J. Callanan, A. Matsuda, A. Mineo, T. Kurosu, and M. Kikuchi, Solid State Commun. 15, 119 (1974).

${ }^{16}$ V. M. Kuz'menko and V. I. Mel'nikov, Zh. Eksp. Teor. Fiz. 82, 802 (1982) [Sov. Phys.-JETP 55, 474 (1982)].

${ }^{17}$ R. B. Gold, J. F. Gibbons, T. J. Magee, J. Peng, R. Ormond, V. R. Deline, and C. A. Evans, in Laser and Electron Beam Processing of Materials, edited by C. W. White and P. S. Peercy (Academic, New York, 1980), p. 221.

18J. C. C. Fan, H. J. Zeiger, R. P. Gale, and R. L. Chapman, Appl. Phys. Lett. 36, 158 (1980).

${ }^{19}$ R. L. Chapman, J. C. C. Fan, H. J. Zeiger, and R. P. Gale, Appl. Phys. Lett. 37, 292 (1980).

${ }^{20}$ H. J. Zeiger, J. C. C. Fan, B. J. Palm, R. P. Gale, and R. L.
Chapman, in Laser and Electron Beam Processing of Materials, Ref. 17, p. 234.

${ }^{21}$ R. L. Chapman, J. C. C. Fan, H. J. Zeiger, and R. P. Gale, in Laser and Electron-Beam Solid Interactions, edited by J. F. Gibbons, L. D. Hess, and T. W. Sigmon (North-Holland, Amsterdam, 1981), p. 81.

${ }^{22}$ H. J. Zeiger, J. C. C. Fan, B. J. Palm, R. L. Chapman, and R. P. Gale, Phys. Rev. B 25, 4002 (1982).

${ }^{23}$ R. A. Lemons and M. A. Bösch, Appl. Phys. Lett. 39, 343 (1981).

${ }^{24}$ G. Auvert, D. Bensahel, A. Georges, V. T. Nguyen, P. Henoc, F. Morin, and P. Coissard, Appl. Phys. Lett. 38, 613 (1981).

${ }^{25}$ G. Auvert, D. Bensahel, A. Perio, V. T. Nguyen, and G. A. Rozgonyi, Appl. Phys. Lett. 39, 724 (1981).

${ }^{26} \mathrm{D}$. Bensahel and G. Auvert, in Laser-Solid Interactions and Transient Thermal Processing of Materials, edited by $\mathrm{J}$. Narayan, W. L. Brown, and R. A. Lemons (North-Holland, Amsterdam, 1983), p. 165.

${ }^{27}$ For a review, see J. M. Poate and W. L. Brown, Physics Today 31(6), 24 (1982).

${ }^{28} \mathrm{G}$. H. Gilmer and H. J. Leamy, in Laser and Electron Beam Processing of Materials, Ref. 17, p. 227.

${ }^{29}$ V. A. Shklovskii, Dokl. Akad. Nauk SSSR 261, 1343 (1981) [Sov. Phys.—Dokl. 26, 1155 (1981)]; Zh. Eksp. Teor. Fiz. 82, 536 (1982) [Sov. Phys._JETP 55, 311 (1982)]; A. V. Koronov and V. A. Shklovskii (unpublished).

${ }^{30}$ W. van Saarloos and J. D. Weeks, Phys. Rev. Lett. 51, 1046 (1983).

${ }^{31}$ W. van Saarloos and J. D. Weeks, in Proceedings of the International Conference on Fronts, Interfaces and Patterns, edited by A. R. Bishop, L. J. Campbell, and P. J. Channell [Physica $\mathrm{D}$ (to be published)].

${ }^{32}$ D. E. Temkin and V. B. Polyakov, Kristallografiya 21, 661 (1976) [Sov. Phys.-Crystallogr. 21, 374 (1976)].

${ }^{33}$ G. L. Olson, S. A. Kokorowski, J. A. Roth, and L. D. Hess, in Laser-Solid Interactions and Transient Thermal Processing of Materials, Ref. 26, p. 141.

${ }^{34}$ These effects are extremely important for complex materials 
like salol (see, e.g., Ref. 35) in which the molecules have to overcome an activation barrier during crystallization. Molecular-dynamics simulations of Lennard-Jones molecules by Broughton et al. (Ref. 36) suggest that there is no activation barrier for simple molecules, but the growth rate still approaches zero for low temperatures because of the slowing down of molecular motion.

${ }^{35}$ K. A. Jackson, D. R. Uhlmann, and J. D. Hunt, J. Cryst. Growth 1, 1 (1967).

36J. Q. Broughton, G. H. Gilmer, and K. A. Jackson, Phys. Rev. Lett. 49, 1496 (1982).

${ }^{37}$ For general reviews, see, e.g., R. F. Sekerka, in Crystal Growth, an Introduction, edited by P. Hartman (NorthHolland, Amsterdam, 1973); J. S. Langer, Rev. Mod. Phys. 52, 1 (1980).

${ }^{38}$ In our analysis, we take, for simplicity, $c$ and other parameters to be equal in the $a$ and $c$ phases; since the heat flow in the $c$ phase is most important, values in the $c$ phase should be taken for comparison with experimental data.

${ }^{39}$ Actually, if the growth rate is always nonzero as in Eq. (2.1), mathematically there is a third steady-state solution, where $T^{b} \simeq T^{0}$ and where the steady-state growth velocity is unobservably small, $V^{b} \sim \exp \left(-Q / T^{0}\right)$. This solution has no physical significance, however, since when $T^{b} \simeq T^{0}$ there will be homogeneous nucleation throughout the sample instead of a single propagating $a-c$ boundary (as is assumed in the model). This third branch of solutions is represented in Fig. 4(b) by points in the lower left-hand corner. When $T^{0}$ is large enough, the lowest intersection depicted in Fig. 1(c) may merge with the third one (not sketched); in Fig. 4(b) this happens at $c_{2}$.

${ }^{40}$ One must keep in mind, however, that the large- $d_{0}$ requirement for straight interfaces may be a shortcoming of the model, since heat loss to the substrate is taken into account in a very crude way using only a phenomenological damping term. We believe that in a more realistic treatment of the heat flow to the substrate the finite- $k$ modes will probably be more suppressed compared to the $k=0$ mode, since the phenomenological damping term tends to underestimate the heat loss at regions bulging forward into the $a$ phase, like point $A$ in Fig. 2. If this effect is in reality more important than the curvature corrections to the growth rate, one would expect to see the $k=0$ mode more often close to the threshold temperature $T^{*}$ for self-sustained growth, where the influence of the heat flow to the substrate will be most pronounced. Thus the parallel undulations seen by Wickersham et al. (Ref. 7) may be related to large velocities (large $d_{0}$ ) or substrate interactions.

${ }^{41}$ This result can be obtained by computing the derivative of the term $q V_{L} G_{\mathrm{SS}}\left(x-x_{\mathrm{SS}}^{b}\right)$ in Eq. (4.5b). Since both profiles drop off with the same exponential for $x$ large, one can also show that $R \simeq 2 T_{L}\left(x^{b}\right) / T_{\mathrm{th}}\left(x^{b}\right)$ for $x^{b}$ large. In this case one again finds that $R$ is typically of order 1 or smaller.

${ }^{42}$ H. J. Leamy, W. L. Brown, G. K. Celler, G. Foti, G. H. Gilmer, and J. C. C. Fan, Appl. Phys. Lett. 38, 137 (1981); in Laser and Electron-Beam Solid Interactions, Ref. 21, p. 89.

43J. M. Poate, in Laser-Solid Interactions and Transient Thermal Processing of Materials, Ref. 26, p. 263.

${ }^{44}$ M. O. Thompson, G. J. Galvin, J. W. Mayer, P. S. Peercy, J. M. Poate, D. C. Jacobson, A. G. Cullis, and N. G. Chew (unpublished).

${ }^{45} \mathrm{Also}$ note that a melting temperature $T_{a}^{M}$ of $a$-Sb of about 600 $\mathrm{K}$ (the boundary temperatures probably reached during crystallization of $a-\mathrm{Sb}$ ) would correspond to a ratio $T_{a}^{M} / T_{c}^{M}$ of about $\frac{2}{3}$. This would be rather low compared to the result $T_{a}^{M} / T_{c}^{M} \geq \frac{4}{5}$ found for $\mathrm{Si}$ and $\mathrm{Ge}$ (see, e.g., J. M. Poate, in Laser-Solid Interactions and Transient Processing of Materials, Ref. 26, p. 263, and D. Turnbull, ibid., p. 131).

${ }^{46}$ H. S. Chen and D. Turnbull, J. Appl. Phys. 40, 4214 (1969).

${ }^{47}$ Thermophysical Properties of Matter (Plenum, New York, 1970), Vol. 4.

${ }^{48}$ See, e.g., P. J. Germain, M. A. Paesler, D. E. Sayers, and K. Zellama, in Laser-Solid Interactions and Transient Thermal Processing of Materials, Ref. 26, p. 135.

${ }^{49}$ C. E. Wickersham, Solid State Commun. 34, 907 (1980).

${ }^{50}$ The difference between the laser intensity $J$ and the induced temperature profile $T_{L}$ was neglected in the analysis of Ref. 22.

${ }^{51}$ S. R. Coriell and R. F. Sekerka, J. Cryst. Growth 61, 499 (1983).

52D. A. Kurtze (unpublished). 